\title{
Exact and inexact scaled models for hot forging
}

\author{
Keith Davey $^{\mathrm{ab}}$, Olga Bylya ${ }^{\mathrm{b}}$, Bhaskaran Krishnamurthy ${ }^{\mathrm{b}}$ \\ ${ }^{a}$ School of Mechanical, Aerospace and Civil Engineering, The University of Manchester, \\ Manchester M13 9PL, UK \\ ${ }^{b}$ Advanced Forming Research Centre, University of Strathclyde, 85 Inchinnan Drive, \\ Inchinnan PA4 9LJ, UK.
}

\begin{abstract}
Scaled experimentation continues to play a significant role in process, product design and testing for metallic components and products but for hot forging in particular is recognized to suffer pronounced scale effects with physical behaviour changing with scale.

This paper is concerned with an assessment of a new scaling approach called finite similitude that has appeared in the recent literature and a new methodology for exact and inexact-experimentation involving scaled experiments. Finite similitude is founded on the scaling of space itself and on a formulation that ensures that the governing physics (in transport form) remain invariant up to proportionality. Unfortunately proportionality breaks down with scale and to account for this careful experimental design is needed.

A question of some importance, which is addressed in this paper, is whether it is possible that physically different materials can exhibit similar mechanical behaviour at certain conditions? These are termed "scaled-material twins" if they are able to match the required material response to some degree of accuracy for those ranges of temperature and strain rates that are representative of forging processes. Presented in the paper is a methodology for selecting scaled-material twins and the quantification of errors involved and its effect on scaled experimentation. Trials with hot disc forgings of different materials and sizes are performed to highlight the difficulties associated with scaling but also to demonstrate that scaled experimentation is possible and if correctly designed offers measurable advantages.
\end{abstract}

KEYWORDS: Metal forming; hot forging; scaled experimentation; finite similitude; scaledmaterial twin. 


\section{Introduction}

Hot large-scale forging represents a particular branch of forging, which is unlikely to be substituted or challenged by emerging new methods (such as additive manufacturing) or displaced by new materials including organic matrix composites in the foreseeable future. Large-scale forged parts remain important for both safety and product capability and for critical and high-temperature applications (Politis et al., 2018, Yang, 2009, Chen and Lin, 2013). Bringing innovation to the hot forging of large scale products is however challenging and consequently the pace of its change is currently moving at a comparatively slower pace when contrasted against other similar manufacturing processes (Hawryluk et al., 2017, Gronostajski et al., 2019, Tian, 2009, Zhang, 2015). The reasons for this are manifold but ultimately it boils down to a size effect; big is more difficult and costly to deal with than small. The problems associated with size however appear at first sight to be totally intractable and it is patently impossible to reduce the size of the required components. This paper focuses on a possible solution that is precisely about size or more appropriately scale and the establishment of a new approach to scaled experimentation. If it were possible to replicate these large processes at a smaller scale, then many of the innovations currently applied and being developed for smaller components could immediately be transferred across.

There are obstacles to scaling however as revealed by the copious pieces of work in the academic literature dealing with the scaling of industrial processes including popular books by Sedov (1993), Zohuri (2015), Barenblatt (2003) and Kline (2012) although only limited work for forging (Altan, et al., 1970 Navarrete et al. 2001, Pawelski, 1992), which confirm that direct scaling is virtually impossible and does not provide a true representation of the mechanics of the process. Despite this apparent terminal limitation, reduced-scaled trials are still being performed in many research/industrial centres around the world. Presently however, there exists no scientifically or practically agreed methodology for interpreting scaled results even in the simplest case of singleoperation manufacturing processes. The question of physical modelling (Sofuoglu and Rasty, 2000, Eckerson and Liechty, 2008, Krishnamurthy et al., 2017, Davey et al., 2017) for a chain of operations has not even been considered and the subject is completely absent from the open literature. The main reason for this is that from the mathematical point of view this problem is far from trivial. There exist few approaches of exact physical scaling (Krishnamurthy et al., 2017) but all are based on the use of different modelling materials; see for example the works of Casaburo et al. (2019), Simitses and Rezaeepazhand (1993) and Coutinho et al. (2016), for structural scaling. For industry however many metallurgical aspects (e.g. microstructure) are themselves targets of a successful forging. From the viewpoint of physical scaling this requirement breaks similitudes, which makes existing scaling approaches inapplicable.

Briefly summarising the current state of the problem is as: 
- There exists previous multiple attempts of physical simulation of single forging operations using soft modelling materials like tin, lead, wax, zinc and with limited scaling ratio (Altan, 1970).

- The principal aim of previous physical trials was limited to particular characteristics of the forging process (e.g. force, geometry) and was not aimed to capture the complete material behaviour (Navarrete et al. 2001, Pawelski, 1992)

- There have been no attempts to simulate the total technological chain (the sequence of forging operations and heat treatments).

- All previous studies used modelling materials (Sofuoglu and Rasty, 2000, Eckerson and Liechty, 2008, Krishnamurthy et al., 2017, Davey, et al., 2017) as per the rules of exact scaling do not give the ability to capture the specifics of metallurgical behaviour of the material and transformation of its microstructure.

A critical part of this paper is the development of a new scaling approach (named inexact scaling) founded on the scaling of space and the application of transport equations. The scaling of space is a radically novel approach to scaling that hitherto has not been considered until very recently (Davey et al. 2017, Al-Tamimi et al., 2018, Moghaddam et al. 2018, Ochoa-Cabrero et al. 2018, Sadeghi et al., 2019a, Sadeghi, 2019b). The idea that space expands and distorts, although not new to physics (e.g. cosmology) its application to physical modelling and especially to real industrial processes is considered new. The scaling concept enables the physics of processes to be projected into a scaled space and vice versa, thus providing quantification of the validity of any physical model, which fortifies a particular weakness in the physical modelling approach. The concept involves a portion of space identified by a control volume (free to move and distort) coupled to a version residing in a scaled space. The processes under investigation are contained within the control volumes and can include die sets, machines and any aspect deemed important to the success of the scaled experiment. The physics in the two spaces are described using transport equations (ten in all for: volume, mass, momentum, movement, energy and entropy) and are deemed to possess finite similitude if found to be proportional in some sense. Finite similitude always exists in continuum mechanics for isotropic scaling (Davey et al. 2017) and this concept is the linchpin to new innovation in forging. Although, parts of the theory are already developed its application to real industrial processes remains a significant challenge due to the constraints imposed by commercial equipment and the requirements for microstructural information. The required utilisation of the actual or scaled-material twins in the scaled models makes the problem particularly challenging and undoubtedly leads to a mismatch between the actual and projected physics. Critical features of a forging process are temperature and strain rate trajectories (thermomechanical history), triaxiality, which are related to the transformation of mechanical properties. Considered in this paper is a systematic analysis of the mismatch that takes 
place and the development of an approach for the approximate restoration of the real process from a set of carefully selected trials, measurements and analysis. A particular novel aspect of the work is the use of finite element analysis for the solution of inverse problems arising from the scaled experimentation. This feature facilitates the establishment of a "digital twin" for the scaled forging process, which can be used to interrogate the relationship to the full scale process.

The practical limitations of finite similitude mean that all the features of full-scale forging processes cannot be exactly replicated in a scaled model. However, exact-finite similitude provides the yardstick by which scaled processes are measured and its limitations are discussed in Section 2. Considered in this section is the classical scaling approach of dimensional analysis, which is contrasted against finite similitude. It is shown that both theories involve proportional physical fields with are assumed a priori for dimensional analysis but arise out of the finite similitude theory. Established is an alternative view of scaling and finite similitude, which is attained if and only if the length scale is removed from all the governing transport equations. The practical applicability of the finite similitude theory for scaled experimentation is discussed in Section 3. The creation of material and forging-digital twins is introduced along with the selection of scaling parameters. An experimental study is performed in Section 4 where the limitations and benefits of scaling are revealed.

\section{Exact scaling and its limitations}

Two universal but closely related methods exist for scaling, which are finite similitude and dimensional analysis. Finite similitude focuses on transport forms and seeks invariance up to a constant of proportionality and considers scaling as a space deformation process. The goal of dimensional analysis however is the invariance of dimensionless equations. Both ultimately yield proportional fields and it is of interest to examine the limitations of the two approaches in brief.

Introduced in the book by Sedov (1993) is the concept of finite similitude, which is founded on the idea that space is free to expand and contract. To achieve this mathematically a differential map (diffeomorphism) is introduced between the trial and physical space. Both spaces are real physical spaces where in one the experiment resides and the process of interest resides in the physical space. The map places points in the trial and physical space in one-to-one correspondence, so it makes no difference whether the mapping is from the trial to the physical or vice versa. The map is assumed to be temporally invariant, i.e. of the form $\boldsymbol{x}_{p s} \mapsto \boldsymbol{x}_{t s}$, which leads to the differential $\boldsymbol{d} \boldsymbol{x}_{t s}=F \cdot \boldsymbol{d} \boldsymbol{x}_{p s}$, which in in the form of suffix notation signifies $d x_{t s}^{i}=F_{j}^{i} d x_{p s}^{j}$, where $F^{i}{ }_{j}=\partial x_{t s}^{i} / \partial x_{p s}^{j}$, where subscripts "ts" and "ps" refer to trial and physical space, respectively. The Jacobean is defined in the usual way as $J=\operatorname{det} F$ and it is convenient to define a linear space scaling parameter $\beta$ to satisfy $\beta^{D}=J$, where $D$ is the topological dimension and is taken to be equal to three here (i.e. $D=3$ ). Time is not 
necessarily assumed to run at the same rate in the two spaces, with the symbol $t_{p s}$ reserved for the physical space and $t_{t s}$ for the trial space.

The next step in the definition of finite similitude is the identification of two regions of space, one in the trial space $\Omega_{t s}^{*}$, and another physical space $\Omega_{p s}^{*}$; both regions are free to move and change shape. The regions play a critical role and facilitate a thermodynamic viewpoint with not only energy and entropy transfers being of interest but momentum and movement also. The idea is to relate the transfers between the two regions of space to establish a definition for finite similitude.

The movement of each region is dictated by means of field velocities $\underline{v}_{p s}^{*}$ and $\underline{v}_{t s}^{*}$, with $\underline{v}_{p s}^{*}=D^{*} \boldsymbol{x}_{p s}^{*} / D^{*} t_{p s}=\partial \boldsymbol{x}_{p s}^{*} /\left.\partial t_{p s}\right|_{\chi}$ and $\underline{v}_{t s}^{*}=D^{*} \boldsymbol{x}_{t s}^{*} / D^{*} t_{t s}=\partial \boldsymbol{x}_{t s}^{*} /\left.\partial t_{t s}\right|_{\chi}$, where $\boldsymbol{x}_{p s}^{*} \in \Omega_{p s}^{*}, \boldsymbol{x}_{t s}^{*} \in \Omega_{t s}^{*}$ and $\boldsymbol{\chi}$ belongs to a reference control volume. It is shown in (Davey et al. 2017) that a map of the form $\boldsymbol{x}_{p s}^{*} \mapsto \boldsymbol{x}_{t s}^{*}$ is required in similitude theory and consequently the differential $\boldsymbol{d} \boldsymbol{x}_{t s}^{*}=F^{*} \cdot \boldsymbol{d} \boldsymbol{x}_{p s}^{*}$ applies. However, in order to reflect the distortion of underlying space through the control volumes it is necessary to set $F^{*}=F$. Note that the process of scaling has been effectively transformed into a deformation process albeit it is space that is being transformed. A relationship of the form $\boldsymbol{d x _ { t s } ^ { * }}=F \cdot \boldsymbol{d} \boldsymbol{x}_{p s}^{*}$ is the common starting point in describing deformation, where in this case an element of $\Omega_{p s}^{*}$ is related to an element of $\Omega_{t s}^{*}$. Although definitions for strains for space are not of interest, what is of particular interest however, are relationships for length, area and volume. These are readily obtained with the aid of Nanson's identities $d V_{t s}^{*}=J d V_{p s}^{*}$ and $\underline{n}_{t s} d \Gamma_{t s}^{*}=J F^{-T} \cdot \underline{n}_{p s} d \Gamma_{p s}^{*}$, where $\underline{n}$ signifies a normal vector on elemental area $d \Gamma^{*}$.

The apparatus is now in place to consider the physics associated with the regions $\Omega_{t s}^{*}$ and $\Omega_{p s}^{*}$, i.e. the rules of continuum thermomechanics. Fundamentally, continuum thermomechanics provides relationships between densities (i.e. per unit volume terms) and fluxes (i.e. per unit area terms) and is described by transport equations of the form (in the physical space):

$$
\frac{D^{*}}{D^{*} t_{p s}} \int_{\Omega_{p s}^{*}} \rho_{p s} \psi_{p s} d V_{p s}^{*}+\int_{\Gamma_{p s}^{*}} \rho_{p s} \psi_{p s}\left(\underline{v}_{p s}-\underline{v}_{p s}^{*}\right) \cdot \underline{n}_{p s} d \Gamma_{p s}^{*}=-\int_{\Gamma_{p s}^{*}} \underline{J}_{p s}^{\psi} \cdot \underline{n}_{p s} d \Gamma_{p s}^{*}+\int_{\Omega_{p s}^{*}} \rho_{p s} b_{p s}^{\psi} d V_{p s}^{*}
$$

which describes the transport of field variable $\psi_{p s}$, where $\rho_{p s}$ is density, $\underline{v}_{p s}$ is material velocity, $\underline{J}_{p s}^{\psi}$ is a flux, $b_{p s}^{\psi}$ is a source term, and where the control volume $\Omega_{p s}^{*}$ is transported with velocity $\underline{v}_{p s}^{*}$ and likewise for the trial space 


$$
\frac{D^{*}}{D^{*} t_{t s}} \int_{\Omega_{t s}^{*}} \rho_{t s} \psi_{t s} d V_{t s}^{*}+\int_{\Gamma_{t s}^{*}} \rho_{t s} \psi_{t s}\left(\underline{v}_{t s}-\underline{v}_{t s}^{*}\right) \cdot \underline{n}_{t s}^{*} d \Gamma_{t s}^{*}=-\int_{\Gamma_{t s}^{*}} \underline{J}_{t s}^{\psi} \cdot \underline{n}_{t s}^{*} d \Gamma_{t s}^{*}+\int_{\Omega_{t s}^{*}} \rho_{t s} b_{t s}^{\psi} d V_{t s}^{*}
$$

where $\Gamma_{t s}^{*}$ is the boundary of $\Omega_{t s}^{*}$.

Equation (1) and (2) are presently independent but can be related on substitution of Nanson's identities, which for isotropic scaling reduce to $d V_{t s}^{*}=\beta^{3} d V_{p s}^{*}$ and $\underline{n}_{t s} d \Gamma_{t s}^{*}=\beta^{2} \underline{n}_{p s} d \Gamma_{p s}^{*}$ since $F=\beta I$, where $I$ is the unit tensor/matrix. Thus on substitution of these identities along with the timedifferential relationship $d t_{t s}=g d t_{p s}$ into Equation (2) gives rise to the transport equation

$$
\begin{aligned}
\frac{1}{g} \frac{D^{*}}{D^{*} t_{p s}} \int_{\Omega_{p s}^{*}} \rho_{t s} \psi_{t s} \beta^{3} d V_{p s}^{*}+\int_{\Gamma_{p s}^{*}} \rho_{t s} \psi_{t s} \beta^{2}\left(\underline{v}_{t s}-\underline{v}_{t s}^{*}\right) \cdot \underline{n}_{p s} d \Gamma_{p s}^{*}= \\
\quad-\int_{\Gamma_{p s}^{*}} \beta^{2} \underline{J}_{t s}^{\psi} \cdot \underline{n}_{p s} d \Gamma_{p s}^{*}+\int_{\Omega_{p s}^{*}} \rho_{t s} \beta^{3} b_{t s}^{\psi} d V_{p s}^{*}
\end{aligned}
$$

where implicit in the notation adopted here is that the control volumes applied in Equations (2) and (3) are identical.

Multiplication of Equation (3) with $g$ and a positive scalar $\alpha^{\psi}$ (since seeking proportional physics), where it is understood that in order for $\alpha^{\psi}$ to pass under the integration signs and through the timederivative it is necessary and sufficient that it is spatially and temporally invariant. Contrasting this equation with Equation (1) provides the following identities:

$$
\begin{aligned}
& \rho_{p s} \psi_{p s}=\alpha^{\psi} \rho_{t s} \beta^{3} \psi_{t s} \\
& \rho_{p s} \psi_{p s}\left(\underline{v}_{p s}-\underline{v}_{p s}^{*}\right)=\alpha^{\psi} \rho_{t s} \beta^{3} \psi_{t s}\left(g \beta^{-1} \underline{v}_{t s}-g \beta^{-1} \underline{v}_{t s}^{*}\right) \\
& \underline{J}_{p s}^{\psi}=\alpha^{\psi} g \beta^{2} \underline{J}_{t s}^{\psi} \\
& \rho_{p s} b_{p s}^{\psi}=g \alpha^{\psi} \rho_{t s} \beta^{3} b_{t s}^{\psi}
\end{aligned}
$$

where comparison of (4a) and (4b) yields $\underline{v}_{p s}-\underline{v}_{p s}^{*}=g \beta^{-1}\left(\underline{v}_{t s}-\underline{v}_{t s}^{*}\right)$.

Equations (4) provide necessary and sufficient conditions for finite similitude and are required to be satisfied for all transport equations. Finite similitude has been confirmed for isotropic scaling and details on this can be found in reference (Davey, 2017) and application details found in works by Davey et al. (2017), Al-Tamimi et al. (2018), Moghaddam et al. (2018), Ochoa-Cabrero et al. (2018), Sadeghi et al. (2019a), Sadeghi, (2019b). Volume conservation provides the identity $\underline{v}_{p s}^{*}=g \beta^{-1} \underline{v}_{t s}^{*}$ (shown below) and consequently $\underline{v}_{p s}=g \beta^{-1} \underline{v}_{t s}$ and a particular point to note is that finite similitude leads ultimately to proportional physical fields, which happens to be the starting point of dimensional analysis. The overall concept of finite similitude is presented in Fig. 1 where a body of mass $\Omega_{p s}^{m}$, 
which is allowed to move and deform and intersects with the control volume $\Omega_{p s}^{*}$. The space-scaling process and its effects are illustrated in the figure, which can be contrasted against a scaled experiment in the trial space. The reference space is where trial and scaled spaced behaviours are compared and depicted in the figure are three tracked-material points along with mismatch in geometry.

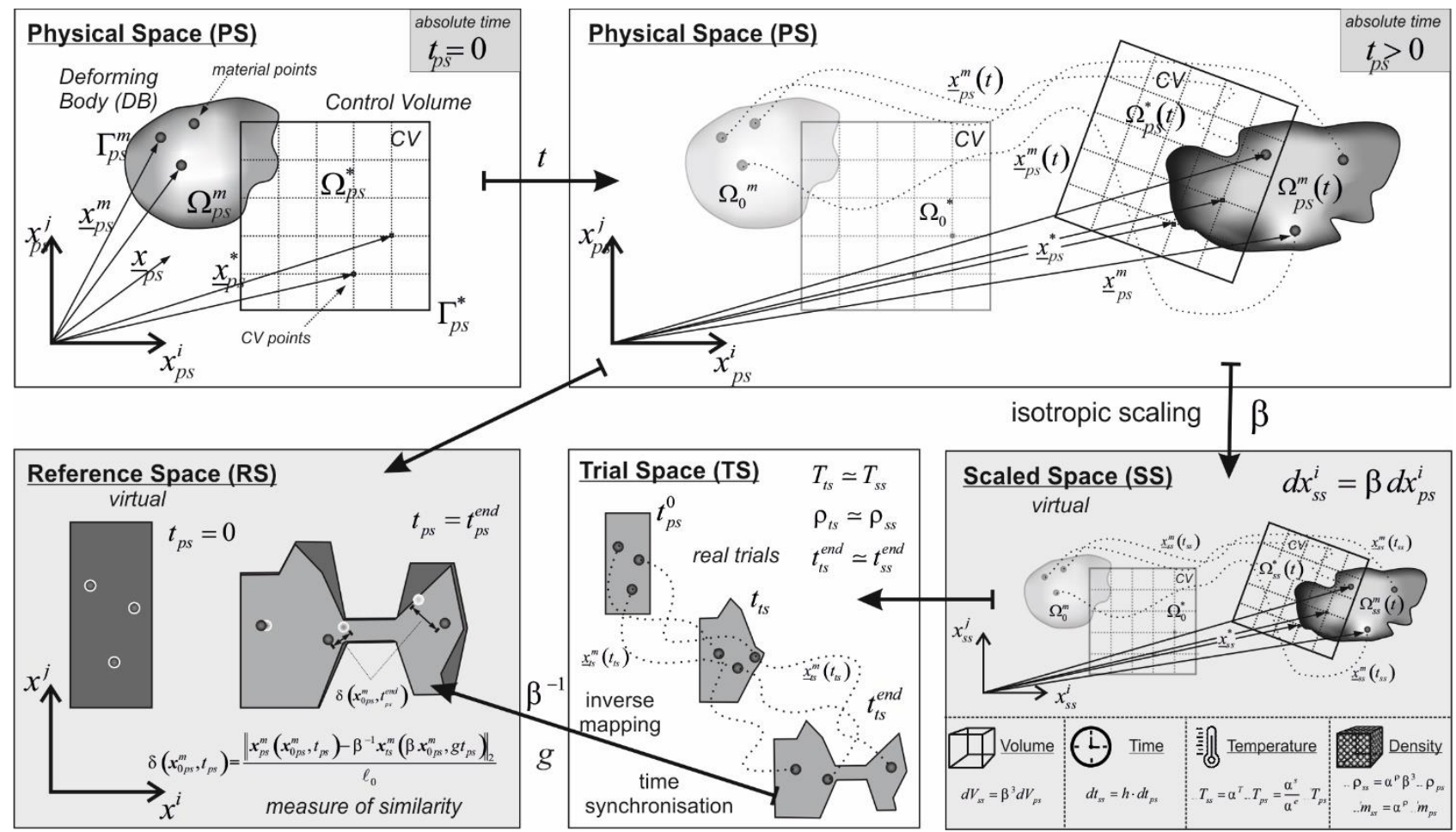

Figure 1. A schematic representation of the overall finite-similitude scaling concept and the methodology adopted for analysis.

A feature of finite similitude and dimensional analysis is proportional fields which for dimensional analysis takes the general form $\mathfrak{I}_{t s}=\xi^{\mathfrak{I}} \mathfrak{I}_{p s}$ and for time, space, velocity and pressure gives: $t_{t s}=\xi^{t} t_{p s}, x_{t s i}=\xi^{x} x_{p s i}, v_{t s i}=\xi^{v} v_{p s i}$ and $p_{t s}=\xi^{p} p_{p s}$. These arise immediately from the assumed invariance of dimensionless physics, where for example $x_{i}=\chi X_{i}$ (with $X_{i}$ dimensionless) leads to $X_{i}=\chi_{t s}^{-1} x_{t s i}=\chi_{p s}^{-1} x_{p s i}$ and consequently $\xi^{x}=\chi_{t s} \chi_{p s}^{-1}$ and similarly for other scalar, vector and tensor fields.

Finite similitude on the other hand does not assume proportional fields a priori and is arrived at by multiplying the transport equations at length-scale $\beta$ by $\alpha^{\psi}$ to arrive at $\alpha^{\psi} \mathrm{T}^{\psi}=0$, where $\mathrm{T}^{\psi}$ is defined in Equation (3) and takes the form

$$
\begin{array}{r}
\mathrm{T}^{\psi}(\beta)=\frac{D^{*}}{D^{*} t_{p s}} \int_{\Omega_{p s}^{*}} \rho_{t s} \psi \psi_{t s} \beta^{3} d V_{p s}^{*}+\int_{\Gamma_{p s}^{*}} \rho_{t s} \psi_{t s} \beta^{2} g\left(\underline{v}_{t s}-\underline{v}_{t s}^{*}\right) \cdot \underline{n}_{p s} d \Gamma_{p s}^{*}+ \\
\int_{\Gamma_{p s}^{*}} \beta^{2} g \underline{J}_{t s}^{\psi} \cdot \underline{n}_{p s} d \Gamma_{p s}^{*}-\int_{\Omega_{p s}^{*}} \rho_{t s} \beta^{3} g b_{t s}^{\psi} d V_{p s}^{*}
\end{array}
$$


where substitution of the similitude conditions $\underline{v}_{p s}=g \beta^{-1} \underline{v}_{t s}$ and $\underline{v}_{p s}^{*}=g \beta^{-1} \underline{v}_{t s}^{*}$ ensures that both material and control volume points are synchronised, respectively and provides

$$
\begin{array}{r}
\mathrm{T}^{\psi}(\beta)=\frac{D^{*}}{D^{*} t_{p s}} \int_{\Omega_{p s}^{*}} \rho_{t s} \psi_{t s} \beta^{3} d V_{p s}^{*}+\int_{\Gamma_{p s}^{*}} \rho_{t s} \psi_{t s} \beta^{3}\left(\underline{v}_{p s}-\underline{v}_{p s}^{*}\right) \cdot \underline{n}_{p s} d \Gamma_{p s}^{*}+ \\
\int_{\Gamma_{p s}^{*}} \beta^{2} g \underline{J}_{t s}^{\psi} \cdot \underline{n}_{p s} d \Gamma_{p s}^{*}-\int_{\Omega_{p s}^{*}} \rho_{t s} \beta^{3} g b_{t s}^{\psi} d V_{p s}^{*}
\end{array}
$$

which also that ensure $\alpha^{\psi} \mathrm{T}^{\psi}=0$ appears in the form of a transport equation in the physical space.

It follows that volume conservation takes the form

$$
\alpha^{1} \mathrm{~T}^{1}(\beta)=\frac{D^{*}}{D^{*} t_{p s}} \int_{\Omega_{p s}^{*}}\left[\alpha^{1} \beta^{3}\right] d V_{p s}^{*}-\int_{\Gamma_{p s}^{*}}\left[\alpha^{1} \beta^{3}\right] \underline{v}_{p s}^{*} \cdot \underline{n}_{p s} d \Gamma_{p s}^{*}=0
$$

which is evidently valid for arbitrary values of $\alpha^{1}$ and $\beta$, and is a consequence of the assumed identity $\underline{v}_{p s}^{*}=g \beta^{-1} \underline{v}_{t s}^{*}$.

Continuity on the other hand takes the form

$$
\alpha^{\rho} \mathrm{T}^{\rho}(\beta)=\frac{D^{*}}{D^{*} t_{p s}} \int_{\Omega_{p s}^{*}}\left[\alpha^{\rho} \rho_{t s} \beta^{3}\right] d V_{p s}^{*}+\int_{\Gamma_{p s}^{*}}\left[\alpha^{\rho} \rho_{t s} \beta^{3}\right]\left(\underline{v}_{p s}-\underline{v}_{p s}^{*}\right) \cdot \underline{n}_{p s} d \Gamma_{p s}^{*}=0
$$

so $\alpha^{\rho} \rho_{t s} \beta^{3}$ can be consider as a form of "density", which on consideration of momentum

$$
\begin{gathered}
\alpha^{v} \mathrm{~T}^{v}(\beta)=\frac{D^{*}}{D^{*} t_{p s}} \int_{\Omega_{p s}^{*}}\left[\alpha^{v} g^{-1} \beta \rho_{t s} \beta^{3}\right] \underline{v}_{p s} d V_{p s}^{*}+\int_{\Gamma_{p s}^{*}}\left[\alpha^{v} g^{-1} \beta \rho_{t s} \beta^{3}\right] \underline{v}_{p s}\left(\underline{v}_{p s}-\underline{v}_{p s}^{*}\right) \cdot \underline{n}_{p s} d \Gamma_{p s}^{*}+ \\
-\int_{\Gamma_{p s}^{*}} \alpha^{v} g \beta^{2} \underline{\underline{\sigma}}_{t s} \cdot \underline{n}_{p s} d \Gamma_{p s}^{*}-\int_{\Omega_{p s}^{*}} \alpha^{v} \rho_{t s} g \beta^{3} \underline{b}_{t s} d V_{p s}^{*}=0
\end{gathered}
$$

gives $\alpha^{v}=\alpha^{\rho} g \beta^{-1}$, which is necessary to reduce the terms in the square brackets to density " $\alpha^{\rho} \rho_{\text {ts }} \beta^{3}$ "; similarly for movement

$$
\begin{gathered}
\alpha^{u} \mathrm{~T}^{u}(\beta)=\frac{D^{*}}{D^{*} t_{p s}} \int_{\Omega_{p s}^{*}}\left[\alpha^{u} \rho_{t s} \beta^{3} \beta\right] \beta^{-1} \underline{u}_{t s} d V_{p s}^{*}+\int_{\Gamma_{p s}^{*}}\left[\alpha^{u} \rho_{t s} \beta^{3} \beta\right] \beta^{-1} \underline{u}_{t s}\left(\underline{v}_{p s}-\underline{v}_{p s}^{*}\right) \cdot \underline{n}_{p s} d \Gamma_{p s}^{*} \\
-\int_{\Omega_{p s}^{*}}\left[\alpha^{u} \beta \rho_{t s} \beta^{3}\right] \underline{v}_{p s} d V_{p s}^{*}=0
\end{gathered}
$$

and the right-hand side provides $\alpha^{u}=\alpha^{\rho} \beta^{-1}$ and the left hand side therefore gives $\underline{u}_{t s}=\beta \underline{u}_{p s}$, again to return density " $\alpha^{\rho} \rho_{t s} \beta^{3}$ " in the square brackets. 
Energy follows a similar pattern and takes the form

$$
\begin{aligned}
& \alpha^{e} \mathbf{T}^{e}(\beta)=\frac{D^{*}}{D^{*} t_{p s}} \int_{\Omega_{p s}^{*}}\left[\alpha^{\rho} \rho_{t s} \beta^{3}\right]\left(\frac{\alpha^{e}}{\alpha^{\rho}}\right) u_{t s}+\left[\alpha^{e} \rho_{t s} \beta^{3} g^{-2} \beta^{2}\right] \frac{v_{p s}^{2}}{2} d V_{p s}^{*} \\
& \quad+\int_{\Gamma_{p s}^{*}}\left[\alpha^{\rho} \rho_{t s} \beta^{3}\right]\left(\frac{\alpha^{e}}{\alpha^{\rho}}\right) u_{t s}\left(\underline{v}_{p s}-\underline{v}_{p s}^{*}\right) \cdot \underline{n}_{p s} d \Gamma_{p s}^{*}+\int_{\Gamma_{p s}^{*}}\left[\alpha^{e} \rho_{t s} \beta^{3} g^{-2} \beta^{2}\right] \frac{v_{p s}^{2}}{2}\left(\underline{v}_{p s}-\underline{v}_{p s}^{*}\right) \cdot \underline{n}_{p s} d \Gamma_{p s}^{*} \\
& \quad+\int_{\Gamma_{p s}^{*}}\left(\alpha^{e} g \beta^{2} \underline{q}_{t s}\right) \cdot \underline{n}_{p s} d \Gamma_{p s}^{*}-\int_{\Gamma_{p s}^{*}}\left(\alpha^{e} g \beta^{2} \underline{v}_{t s} \cdot \underline{\underline{\sigma}}_{t s}\right) \cdot \underline{n}_{p s} d \Gamma_{p s}^{*} \\
& \quad-\int_{\Omega_{p s}^{*}}\left[\alpha^{\rho} \rho_{t s} \beta^{3}\right]\left(\frac{\alpha^{e}}{\alpha^{\rho}}\right) g Q_{t s} d V_{p s}-\int_{\Omega_{p s}^{*}}\left[\alpha^{\rho} \rho_{t s} \beta^{3}\right]\left(\frac{\alpha^{e}}{\alpha^{\rho}}\right) g \underline{b}_{t s} \cdot \underline{v}_{t s} d V_{p s}^{*}=0
\end{aligned}
$$

which infers $\alpha^{e}=\alpha^{\rho} g^{2} \beta^{-2}$ and similarly for entropy

$$
\begin{gathered}
\alpha^{s} \mathbf{T}^{s}(\beta)=\frac{D^{*}}{D^{*} t_{p s}} \int_{\Omega_{p s}^{*}}\left[\alpha^{\rho} \rho_{t s} \beta^{3}\right]\left(\frac{\alpha^{s}}{\alpha^{\rho}}\right) s_{t s} d V_{p s}+\int_{\Gamma_{p s}^{*}}\left[\alpha^{\rho} \rho_{t s} \beta^{3}\right]\left(\frac{\alpha^{s}}{\alpha^{\rho}}\right) s_{t s}\left(\underline{v}_{p s}-\underline{v}_{p s}^{*}\right) \cdot \underline{n}_{p s} d \Gamma_{p s}^{*} \\
+\int_{\Gamma_{p s}^{*}}\left(\frac{\alpha^{s}}{\alpha^{e}}\right) T_{t s}^{-1}\left(\alpha^{e} g \beta^{2} \underline{q}_{t s}\right) \cdot \underline{n}_{p s} d \Gamma_{p s}^{*}-\int_{\Omega_{p s}^{*}}\left(\frac{\alpha^{s}}{\alpha^{e}}\right) T_{t s}^{-1}\left[\alpha^{\rho} \rho_{t s} \beta^{3}\right]\left(\frac{\alpha^{e}}{\alpha^{\rho}}\right) g Q_{t s} d V_{p s} \\
-\int_{\Omega_{p s}^{*}} \alpha^{s} g \beta^{3} \dot{s}_{t s} d V_{p s}^{*}=0
\end{gathered}
$$

where the temperature identity $\alpha^{s} T_{p s}=\alpha^{e} T_{t s}$ is revealed on examination of heat and entropy flux terms in this and Equation (11).

Note that the identities $\alpha^{v}=\alpha^{\rho} g \beta^{-1}, \alpha^{u}=\alpha^{\rho} \beta^{-1}, \underline{u}_{t s}=\beta \underline{u}_{p s}, \alpha^{e}=\alpha^{\rho} g^{2} \beta^{-2}$ all stem from the similarity condition $\underline{v}_{p s}=g \beta^{-1} \underline{v}_{t s}$ (with $\underline{v}_{p s}^{*}=g \beta^{-1} \underline{v}_{t s}^{*}$ assumed) but the identity $\alpha^{s} T_{p s}=\alpha^{e} T_{t s}$ is a manifestation of a product (i.e. $\underline{q}_{t s}$ and $T_{t s}^{-1}$ ) appearing in the entropy equation. An alternative viewpoint however is that the role of these identities is to remove the length scalar $\beta$ from the transport equations, thus making them valid at any scale. Finite similitude in this sense is achieved if and only if $\beta$ is removable from all the transport equations.

Dimensional analysis assumes implicitly that the dimensionless governing equations do not change with scale. This aspect is explicit with the transport equations, where the dependence on $\beta$ is more overt. Finite similitude is definable as

$\frac{d}{d \beta}\left[\alpha^{\psi}(\beta) \mathrm{T}^{\psi}(\beta)\right] \equiv 0$ 
where the equality symbol " $\equiv "$ is used to indicate that equality applies to all the integrands in the transport equation $\alpha^{\psi} \mathrm{T}^{\psi}=0$.

Finite similitude is simply $d\left[\alpha^{\psi} \mathrm{T}^{\psi}\right] / d \beta \equiv 0, \forall \beta>0$, which infers that $\alpha^{\psi} \mathrm{T}^{\psi}$ is invariant of $\beta$, so $\quad \alpha^{\psi}(1) \mathrm{T}^{\psi}(1) \equiv \alpha^{\psi}(\beta) \mathrm{T}^{\psi}(\beta)$, with $\alpha^{\psi}(1)=1$, i.e. corresponding integrands in $\mathrm{T}^{\psi}(1)$ and $\alpha^{\psi}(\beta) \mathrm{T}^{\psi}(\beta)$ match. The integrands in $\alpha^{\psi} \mathrm{T}^{\psi}$ are required to be independent of $\beta$ to satisfy the equality $\mathbf{T}_{p s}^{\psi} \equiv \alpha^{\psi} \mathbf{T}_{t s}^{\psi}$ and leads to the finite similitude relationships:

scaling parameters: $\left\{\alpha^{1}=\beta^{-3}, \alpha^{v}=g \beta^{-1} \alpha^{\rho}, \alpha^{u}=\beta^{-1} \alpha^{\rho}, \alpha^{e}=g^{2} \beta^{-2} \alpha^{\rho}\right\}$;

field variables: $\left\{\rho_{p s}=\alpha^{\rho} \beta^{3} \rho_{t s}, \underline{v}_{p s}=g \beta^{-1} \underline{v}_{t s}, \underline{u}_{p s}=\beta^{-1} \underline{u}_{t s}, u_{p s}=g^{2} \beta^{-2} u_{t s}, \alpha^{s} T_{p s}=\alpha^{e} T_{t s}\right\}$;

transfer identities: $\left\{\underline{\underline{\sigma}}_{p s}=\alpha^{v} g \beta^{2} \underline{\underline{\sigma}}_{t s}, \underline{q}_{p s}=\alpha^{e} g \beta^{2} \underline{q}_{t s}\right\}$ and

source identities: $\left\{\rho_{p s} Q_{p s}=\alpha^{e} g \beta^{3} \rho_{t s} Q_{t s}, \rho_{p s} \underline{b}_{p s}=\alpha^{v} g \beta^{3} \rho_{t s} \underline{b}_{t s}, \dot{s}_{p s}=\alpha^{s} g \beta^{3} \dot{s}_{t s}\right\}$

The details on this can be found in reference (Davey et al., 2017) although these are readily obtained on applying the identity $\mathrm{T}^{\psi}(1) \equiv \alpha^{\psi}(\beta) \mathrm{T}^{\psi}(\beta)$ to transport equations (7) to (12).

\section{Assessment of the practical applicability of the Theory of Exact Scaling}

This section examines practical measures of similitude that can be readily adopted in finite element simulations and experiments.

\subsection{Method of Analysis of Scaled Experiments}

\subsubsection{The measure of finite similitude}

This section is devoted to the investigation of the practical applicability of the theory of exact scaling in the case of hot forging processes. It is assumed that there is a main real full-scale forging process (real forging) in a physical space (ps) and a scaled model of this process performed in a trial space (ts). The ultimate aim of physical modelling is to achieve exact similitude of these two processes.

To proceed, it is necessary to formulate a means to understand similitude practically and define measures of similarity that can be examined in tests and simulations conducted. The theory presented in Section 2 is founded on behaviours in synchronised regions of space (control volumes) but practical measures in forging invariably involve identifying mass movement. It is convenient therefore to adopt a Lagrangian frame of reference to describe the motion of material. A material point in the physical space $\boldsymbol{x}_{p s}^{m}$ satisfies the material derivative identity $D \boldsymbol{x}_{p s}^{m} / D t_{p s}=\underline{v}_{p s}$ and similarly in the trial 
space $D \boldsymbol{x}_{t s}^{m} / D t_{t s}=\underline{v}_{t s}$. Each of these identities can be integrated to produce a function of the form $\boldsymbol{x}_{0 p s}^{m} \mapsto \boldsymbol{x}_{p s}^{m}\left(\boldsymbol{x}_{0 p s}^{m}, t_{p s}\right)$ (and $\boldsymbol{x}_{0 t s}^{m} \mapsto \boldsymbol{x}_{t s}^{m}\left(\boldsymbol{x}_{0 t s}^{m}, t_{t s}\right)$ ), which is a map of the form $\Omega_{0_{p s}}^{m} \rightarrow \Omega_{t_{p s}}^{m}$ (and $\Omega_{0_{t s}}^{m} \rightarrow \Omega_{t_{t s}}^{m}$ ), where $\Omega_{0_{p s}}^{m}$ and $\Omega_{0_{t s}}^{m}$ are the initial preforms in their respective spaces, see Fig. 1. The domains $\Omega_{t_{p s}}^{m}$ and $\Omega_{t_{t s}}^{m}$ represent the forgings at times $t_{p s}$ and $t_{t s}$, respectively, and by definition are sets of materials points (i.e. continua). Similarity of material points follows immediately from the movement equation and the displacement relationship $\underline{u}_{p s}=\beta^{-1} \underline{u}_{t s}$, where in a Lagrangian framework displacement can be defined in the physical space to be $\underline{u}_{p s}=\boldsymbol{x}_{p s}^{m}-\boldsymbol{x}_{0 p s}^{m}$ and likewise in the trial space $\underline{u}_{t s}=\boldsymbol{x}_{t s}^{m}-\boldsymbol{x}_{0 t s}^{m}$. It immediately follows that similarity for material points requires

$\boldsymbol{x}_{p s}^{m}\left(\boldsymbol{x}_{0 p s}^{m}, t_{p s}\right)=\boldsymbol{x}_{p s}^{m}\left(\beta^{-1} \boldsymbol{x}_{0 t s}^{m}, g^{-1} t_{t s}\right)=\beta^{-1} \boldsymbol{x}_{t s}^{m}\left(\boldsymbol{x}_{0 t s}^{m}, t_{t s}\right)$

or

$\boldsymbol{x}_{t s}^{m}\left(\boldsymbol{x}_{0 t s}^{m}, t_{t s}\right)=\boldsymbol{x}_{t s}^{m}\left(\beta \boldsymbol{x}_{0 p s}^{m}, g t_{p s}\right)=\beta \boldsymbol{x}_{p s}^{m}\left(\boldsymbol{x}_{0 p s}^{m}, t_{p s}\right)$

which gives rise to a measure of local similarity for material points and in terms of Euclidean distance is

$\delta\left(\boldsymbol{x}_{p s}^{m}, \boldsymbol{x}_{t s}^{m}\right)=\frac{\left\|\boldsymbol{x}_{p s}^{m}-\beta^{-1} \boldsymbol{x}_{t s}^{m}\right\|_{2}}{\ell_{0}}$

or equivalently in Lagrangian form for any instant of the forging process

$\delta\left(\boldsymbol{x}_{0 p s}^{m}, t_{p s}\right)=\frac{\left\|\boldsymbol{x}_{p s}^{m}\left(\boldsymbol{x}_{0 p s}^{m}, t_{p s}\right)-\beta^{-1} \boldsymbol{x}_{t s}^{m}\left(\beta \boldsymbol{x}_{0 p s}^{m}, g t_{p s}\right)\right\|_{2}}{\ell_{0}}$

where $\ell_{0}$ is some characteristic normalizing length included to make the measure dimensionless.

This similarity is local, in the sense that $\delta$ can take up different values at different points of the material body and at different instances of time. If full similitude exists between two processes, then $\delta\left(\boldsymbol{x}_{0 p s}^{m}, t_{p s}\right)=0$ for all materials points $\boldsymbol{x}_{0 p s}^{m} \in \Omega_{0 p s}^{m}$ and for instances of time $t_{p s} \in\left[0, t_{p s}^{*}\right]$, where $t_{p s}^{*}$ is the forging time. If on the other hand $\delta\left(\boldsymbol{x}_{0 p s}^{m}, t_{p s}\right) \neq 0$ for even a single point $\boldsymbol{x}_{0 p s}^{m} \in \Omega_{0 p s}^{m}$ or instance of time, then finite similitude is lost.

Local measures of this type naturally give rise to global measures and two possibilities are:

$$
\Delta_{1}=\max _{\substack{x_{p s}^{m} \in \Omega_{0 p s}^{m} \\ t_{p s} \in\left[0, t_{p s}^{m}\right.}} \delta\left(\boldsymbol{x}_{0 p s}^{m}, t_{p s}\right)
$$


and

$$
\Delta_{2}=\max _{x_{0 p s}^{m} \in \Omega_{0 p s}^{m}} \delta\left(\boldsymbol{x}_{0 p s}^{m}, t_{p s}^{*}\right)
$$

which are useful practical measures for contrasting similarity in processed FE simulation results and note that $\Delta_{2} \leq \Delta_{1}$.

Due to the utilisation of remeshing in finite element simulation involving finite deformation, meshes in the physical and trial spaces will invariably be different, but a set of tracking points can be set at the beginning of the process and then analysed at any time step (see reference space in Fig. 1). The situation with the physical (live) trials is more difficult. In hot forging, due to high temperatures and the very large deformations involved, there is no direct ability to mark internal points and trace them. It is sometimes possible to observe flow lines after cutting and polishing, but even in this case the correspondence between points may not be evident (particularly in different materials). The surface of the forged parts can be practically suitable for direct metrological analysis but again because the correspondence between points may not be clear it tends to be limited to an area-to-area comparison.

\subsubsection{The concept of Digital Twins}

An alternative approach is presented in this work, which is based on a combination of numerical and physical trials. The main idea of it is the creation of "digital twins" of the actual forgings and then performing further manipulations with them.

Utilisation of industrial-scale equipment always involves a certain level of variation of boundary conditions. It is normal for these processes that the loading speed, temperatures and friction conditions deviate within 5-10\%; this presents difficulties in examining the repeatability and stability of an operation, and comparison of different forging regimes. Digital twins of the trials are designed to overcome these difficulties and provide a systematic approach for comparison. Figure 2 shows the logic of the development of the digital twin. First, the forging trial is conducted as close as possible to the desired conditions. Actual forging conditions measured during the forging (actual die and air temperatures, time of transfer of the pre-heated preform, time of resting on the dies and actual velocity of the press) are converted into the boundary conditions of the FE model and the process is simulated. Any FE model has certain parameters that cannot be calibrated with absolute accuracy in advance. Friction and heat transfer during material flow on complex die geometry, material flow curves at large deformation and different triaxiality are the most important parts of the model for which extrapolated data of questionable relevance is used. This is the main reason why direct predictive modelling of large-scale forgings can be unreliable. To create an adequate digital twin, the initial FE model of the process is tuned to match the metrology data available from the trial. In this paper, four independent sources of data were used. Thermocouples welded to the inside of the preforms and external thermal 
cameras provide the data for the reconstruction of temperature distribution inside the part. Its comparison with the simulation results helps to tune the (i) thermal conductivities of the forged material and dies, (ii) heat transfer coefficient without and with loading, and (iii) emissivity and heat exchange with the air. After the thermal part of the model is tuned, the simulation is repeated (to ensure that the thermal settings do not contribute to the output error significantly). Then, the obtained geometrical shape is compared to the 3D digital scan of the formed part using GOM inspect software and the flow lines in the simulation are compared to grain-flow lines visible at the polished and etched section of the forged part for adjusting the friction between material and dies through the process. Grain flow lines along with the readings of the press load at every instant of the forging process are finally used for finding an appropriate extrapolation of the equivalent flow stress curves (or calibration of the analytical model) of forged material. If the accuracy of the digital twin is critical, additional forging trials with intermediate stops can be conducted for further refinement of the model.

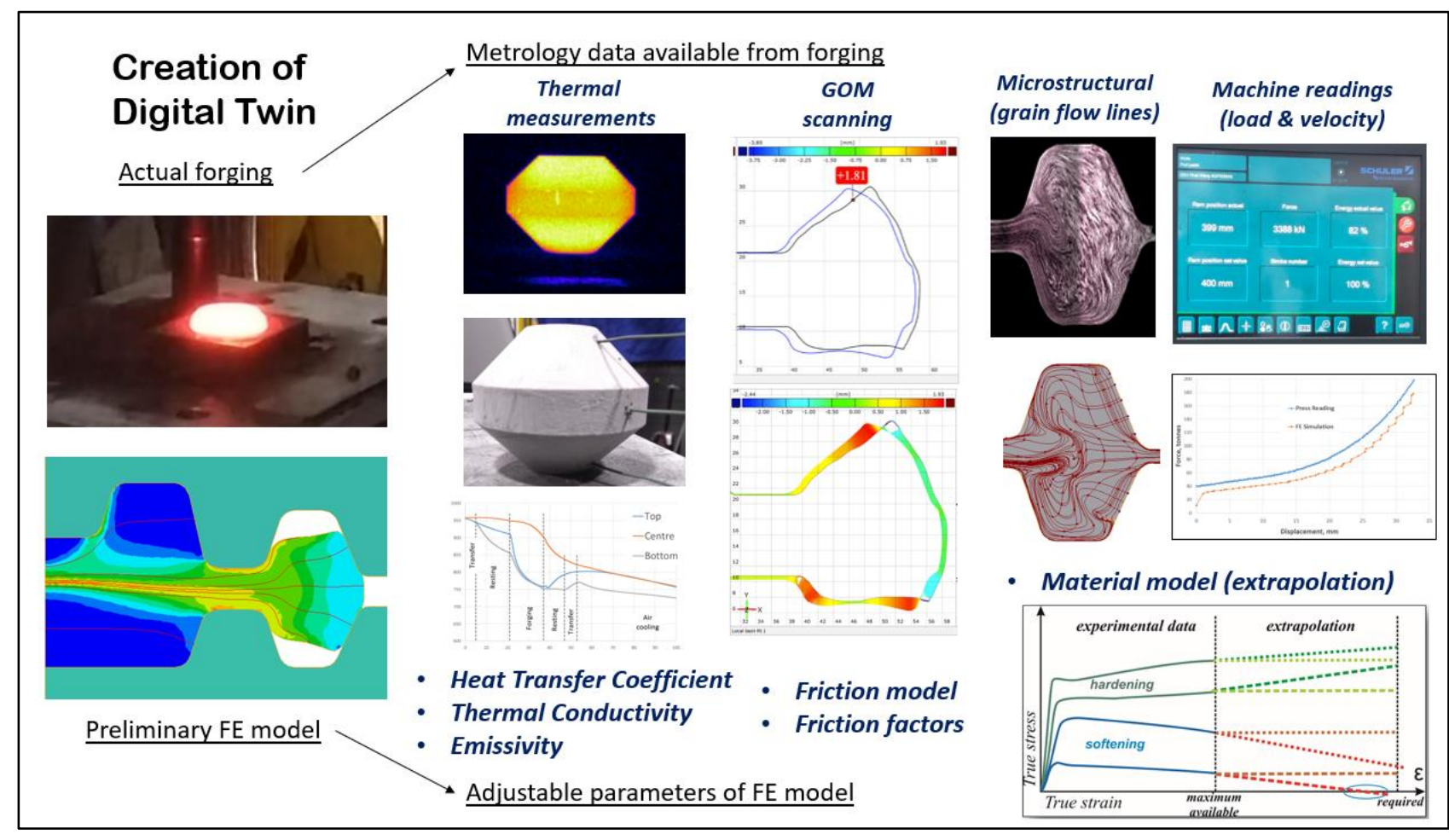

Figure 2. Methodology adopted for the creation of digital twin of the forging process.

The principle scheme of utilisation of digital twins in the analysis of scaling experiments is as follows: first, all scaled experiments are performed as close to the desired conditions. Digital twins of all trials are created as described above. Then, a unified set of simulations is performed using digital twins: same time and mesh settings, exact boundary conditions imposed by scaling theory, same location of tracking points and lines, synchronisation of time, etc. The results of these simulations are now suitable for a comparative analysis. Thanks to the fact that the digital twins provide already digitized data, comparison can be performed not only qualitatively, but quantitatively using all available mathematical and statistical approaches. Besides this, not only the external outputs can be analysed, 
but also the internal points in different parts of preform can be tracked and their thermomechanical histories, displacements, triaxiality etc. can be compared for both scaled and original experiments.

It should be borne in mind that the overall aim here is to devise a means to communicate information from the trial to the physical space and vice versa and to validate this. Many of the important physical quantities from the trials are not measurable however (e.g. stress and heat transfer) and for the results that can be measured (e.g. geometrical aspects) the scalars $\left\{\alpha^{\rho}, \alpha^{v}, \alpha^{u}, \alpha^{e}\right\}$ tend not to be involved (see Section 2.1). Digital twins do facilitate comparisons of the non-measurable quantities but in this work greater importance is placed on results that are measurable and obtained from the experiments.

\subsection{Design of Scaled Experiments - General Methodology}

This section examines the Design of Experiments (DoE) for physical scaled trials. The set of the scaling equations presented in Section 2.1 provide the main guidance for shaping of the scaled down twin process in the trial space. This task splits into two main problems - selection of the modelling material (termed scaled-material twin here) and selection of the scaled forging conditions.

Although on first inspection the DoE procedure appears relatively straightforward, the lack of exact similitude poses some difficulty. Six scalar scaling factors are involved which are $\alpha^{1}$ (volume), $\alpha^{\rho}$ (mass), $\alpha^{v}$ (momentum), $\alpha^{u}$ (movement), $\alpha^{e}$ (energy) and $\alpha^{s}$ (entropy) along with two additional scaling parameters for space $\beta$ and time $g$. Not all scaling parameters are independent as revealed in Section 2.1 and the following relationships hold: $\alpha^{1}=\beta^{-3}, \alpha^{v}=g \beta^{-1} \alpha^{\rho}, \alpha^{u}=\beta^{-1} \alpha^{\rho}$ and $\alpha^{e}=g^{2} \beta^{-2} \alpha^{\rho}$. The dependency arises out of the coupled physics and is a necessary although not sufficient requirement for the elimination of $\beta$ from the transport equations. With the restriction that $\xi^{T}=\alpha^{s} / \alpha^{e}$ (see Fig. 1) there are effectively four independent scaling parameters, which have to be chosen. The length scaling parameter $\beta$ dictates the size of the miniaturised forging and can be chosen (for example) with the capacity of the available forging equipment in mind. The time scaling and temperature scaling parameters $g$ and $\xi^{T}$ temperature scaling parameter define the main constraints of speed and temperature. This leaves the mass scaling parameter $\alpha^{\rho}$, which influences inertial behaviour. However, under the assumption that the total weight of the original (large scale) material is significantly smaller than the load required for its plastic deformation (yield stress times the characteristic area) and metal flow can be treated as quasi-static, inertial effects can be neglected. Then the use of $\alpha^{\rho}$ to satisfy the inertial identity $\rho_{p s}=\alpha^{\rho} \beta^{3} \rho_{t s}$ to high accuracy is not required as any lack of proportionality will not significantly affect the results of the forgings. Note that this assumption is very important for the practical use of scaled experiments and has the effect of freeing up the parameter $\alpha^{\rho}$ for matching elsewhere. It is important to note that the range of the densities 
of the commonly used metallic materials (and solids in general) is quite limited. Thus in a situation where the scaling factor $\beta$ is small (e.g. $10^{-1}, 10^{-2}, 10^{-3}$ ), then there is little scope to obtain a similar order of mass in the original and scaled down processes, and hence a correctly calculated value of $\alpha^{\rho}$ (i.e. satisfying $\rho_{p s}=\alpha^{\rho} \beta^{3} \rho_{t s}$ ) for existing materials can provide rather large values (e.g. $\sim 10^{3}$, $\sim 10^{6}, \sim 10^{9}$ ). With $\rho_{p s}=\rho_{t s}$ for example, exact finite similitude requires $\alpha^{\rho}=\beta^{-3}$, thus limiting $\beta$ or imposing a material with properties that may not exist.

A similar type of problem is also faced in selection of the temperature scaling parameter $\xi^{T}$. If the forging process is isothermal, then the problem of achieving proportionality of temperatures is not so difficult. Unfortunately, the majority of the hot forging processes are not isothermal and it is recognised that the isothermal forging of huge components is rather costly. Involved in hot forging processes are different independent characteristic values of temperature, i.e. the ambient air temperature $T^{\text {amp }}$ (typically around $300 \mathrm{~K}$ ), dies temperatures $T^{\text {tools }}$ and material pre-heat $T^{\text {mat }}$ (Kline, 2012). The initial die temperatures depend on the specification of the forging equipment and can have different ranges of variation; $293 \mathrm{~K}$ to $503 \mathrm{~K}$ is an approximate range for $T^{\text {tools }}$. Material preheat $T^{\text {mat }}$ has the broadest range of temperature variation with $\mathrm{Al}$ alloys being forged at about $600 \mathrm{~K}$ and $\mathrm{Ni}$ alloys requiring $1300 \mathrm{~K}$ plus. Forging temperature normally depends on the specific metallographic behaviour of each material. Important temperatures are $T^{\mathrm{rec}}, T^{\mathrm{pt}}$ and $T^{\mathrm{ps}}$ associated with recrystallization, phase transformations and precipitation; melting temperature $T^{\text {melt }}$ is also an important consideration. Examples of these temperatures are provided in the Table 1, where it is readily evident that all these temperatures and ranges of their variations are non-proportional. Consequently the matching the three equations $T_{t s}^{\mathrm{amp}}=\xi^{T} T_{p s}^{\mathrm{amp}}, T_{t s}^{\mathrm{tools}}=\xi^{T} T_{p s}^{\mathrm{tools}}$ and $T_{t s}^{\mathrm{mat}}=\xi^{T} T_{p s}^{\mathrm{mat}}$ (with the same value of $\xi^{T}$ ) is problematic and more generally the similitude rule $T_{t s}=\xi^{T} T_{p s}$ will be not satisfied. Unlike the mass scaling parameter $\alpha^{\rho}$ the effect of breaking the similitude rule $T_{t s}=\xi^{T} T_{p s}$ is not immediately obvious and quantifiable. This aspect is investigated further in the trials presented below along with strategies to mitigate its effect.

Another feature of similitude is time scaling with the associated scaling parameter $g$. Bearing in mind the issue with ambient and tool temperatures, it might be anticipated that scaled down experiments for a scaled forging with relatively small volume to surface area ratio will be chilled at the surface more than the original larger forging. A possibly strategy for counteracting this chilling effect might be the acceleration of the process with a suitable choice for $g$. There exists unfortunately another important aspect that must be taken into consideration. 
One of the features of the finite similitude approach as presented in Section 2 is that it does not explicitly involve constitutive equations. Constitutive equations are generally required to close the system of transport equations and are related through the transfer identities presented in Section 2, i.e. $\underline{\sigma}_{p s}=\alpha^{v} g \beta^{2} \underline{\sigma}_{t s}$ for work and $\underline{q}_{p s}=\alpha^{e} g \beta^{2} \underline{q}_{t s}$ for heat. Observe that three of the four independent scaling parameters (i.e. $\beta, g, \alpha^{\rho}$ and $\xi^{T}$ ) determine the scaling parameter for stress, which takes the form $\underline{\underline{\sigma}}_{p s}=\xi^{\sigma} \underline{\underline{\sigma}}_{t s}$, where $\xi^{\sigma}=g^{2} \beta \alpha^{\rho}$ since $\alpha^{v}=g \beta^{-1} \alpha^{\rho}$. This informs or provides constraint on the choice of the scaled-material twin. Traditionally, thermomechanical constitutive equations used in hot forging take the multiplicative form

$$
\bar{\sigma}=f(\bar{\varepsilon}, \dot{\bar{\varepsilon}}, T)=\varphi(\bar{\varepsilon}) \phi(\dot{\bar{\varepsilon}}) \chi(T)
$$

where $\bar{\varepsilon}$ is accumulated plastic strain, $\bar{\sigma}$ is equivalent stress and $\dot{\bar{\varepsilon}}$ is strain rate.

An example of this type of equation is the Hansel-Spittel approximation, which is often used in hot forging problems and explicitly takes the form

$$
\sigma=A \bar{\varepsilon}^{n}\left(\frac{\dot{\bar{\varepsilon}}}{\dot{\varepsilon}_{0}}\right)^{m} \exp \left(-\frac{Q}{R T}\right)
$$

and consequently the following relationship applies

$$
\sigma_{p s}=A_{p s} \bar{\varepsilon}_{p s}^{n_{p s}}\left(\frac{\dot{\bar{\varepsilon}}_{p s}}{\dot{\varepsilon}_{0, p y}}\right)^{m_{p s}} \exp \left(-\frac{Q_{p s}}{R T_{p s}}\right)=A_{p s} \bar{\varepsilon}_{p s}^{n_{p s}}\left(\frac{g \dot{\bar{\varepsilon}}_{t s}}{g \dot{\varepsilon}_{0, t s}}\right)^{m_{p s}} \exp \left(-\frac{\alpha^{T} Q_{p s}}{R T_{t s}}\right)=\xi^{\sigma} \sigma^{p s}
$$

which impose the following requirements for exact similitude:

$$
n_{t s}=n_{p s}, m_{t s}=m_{p s}, Q_{t s}=\xi^{T} Q_{p s}, A_{p s}=\xi^{\sigma} A_{t s}
$$

In other words, scaled material twins should have same strain hardening $n$, same strain rate sensitivity $m$ and thermal sensitivity $Q$ satisfying the relationship in Eq. (20) along with proportional magnitudes of stresses with a constant of proportionality equal to $\xi^{\sigma}$. In general, these requirements are obviously not satisfied for the full range of possible temperatures, strain rates and strains. However, what might exist is a "window" where requirements presented in Equation (20) are satisfied to a reasonable degree of accuracy.

One of the best known successes for the selection of matching "window" is in the physical modelling of the processes of superplastic forming (Lin, 2003, Alaborta et al., 2015), conducted in temperature range of $0.5 T^{m}-0.8 T^{m}$, strain rate range of $10^{-4}-10^{-2} \mathrm{~s}^{-1}$, for two-phase fine-grained materials, where $T^{m}=T / T^{\text {melt }}$. These processes are normally almost isothermal, so thermal sensitivity does not 
play any role and requirement of its proportionality can therefore be neglected. The material behaves like an ideal plastic one (without any hardening or softening), so $n=0$ and there is no dependence on strain. Because of this it is sufficient to find two materials with similar strain rate sensitivities. However, even for this case the number of scaled-material twins is limited, and one of the most successful is the couple Ti6Al4V and $\mathrm{Pb}$, which facilitated the study of superplastic flow of Ti6Al4V at high temperatures using $\mathrm{Pb}$ at room temperature (Padmanabhan et al., 2001).

In the case of hot forging, the situation is more complicated since the majority of common forging processes are not isothermal. Material cooling in the areas close to the surface along with adiabatic heating in the zones of active (intensive) plastic deformation develops significantly non-uniform temperature distribution across the deformable body. The complex geometry in close-die forging often leads to flow localizations, thereby development of areas with low and high strain rates. As a result, temperature and strain rate in different material points can have very complicated histories and relatively broad ranges of variation, as shown in Fig. 3(a-c). This makes material selection and detection of a matching window a nontrivial exercise.

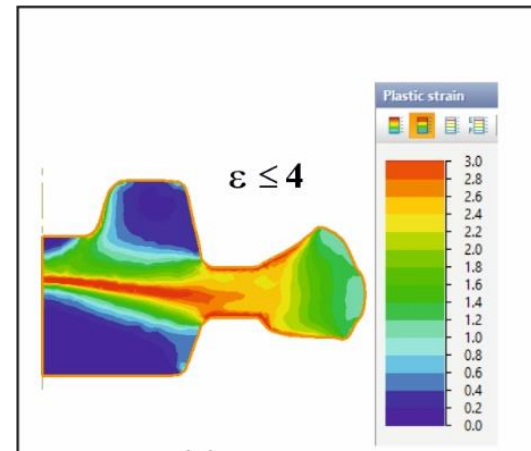

(a)

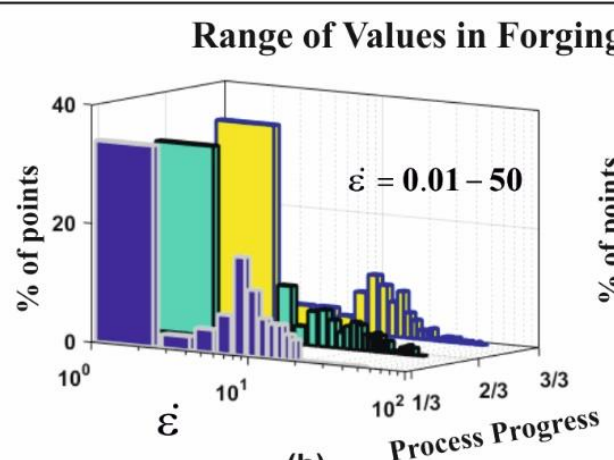

(b)

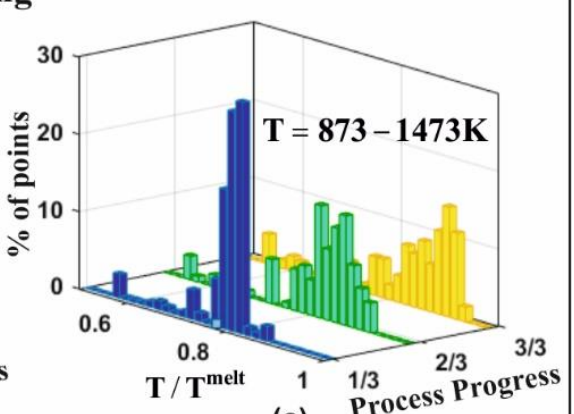

(c)

Determination of "matching window"
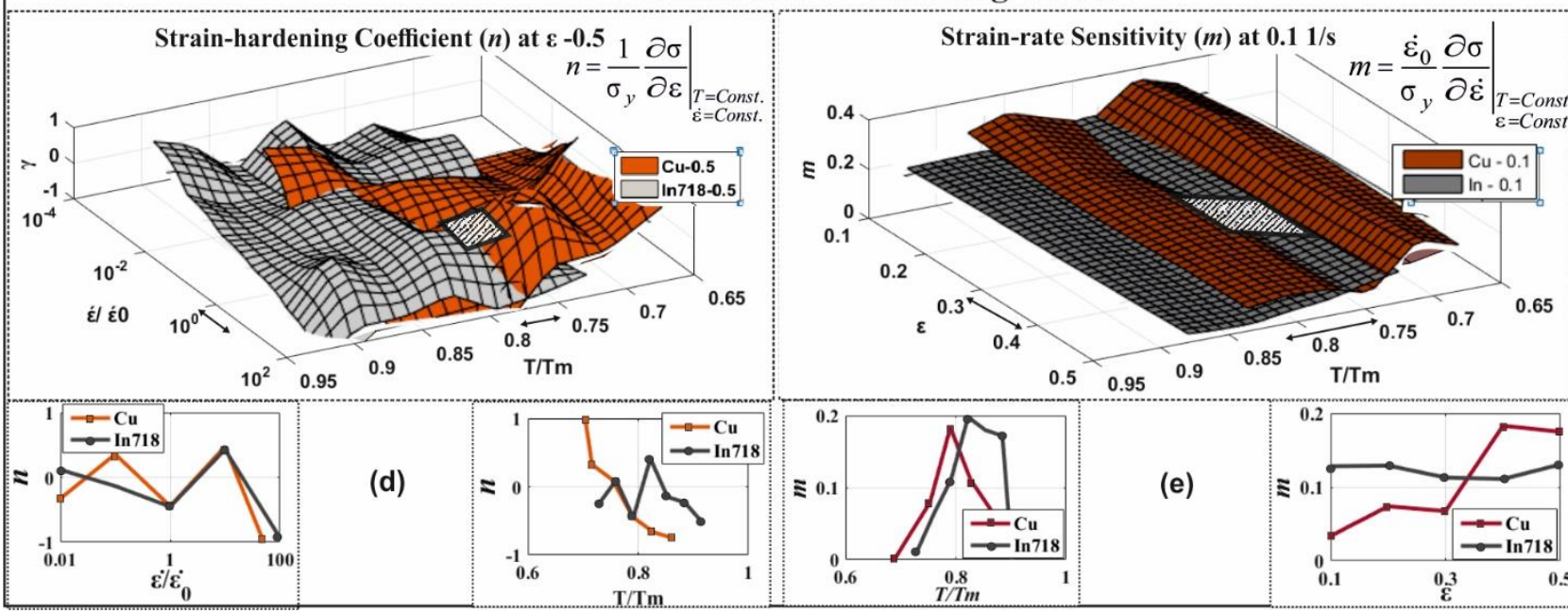

(d)
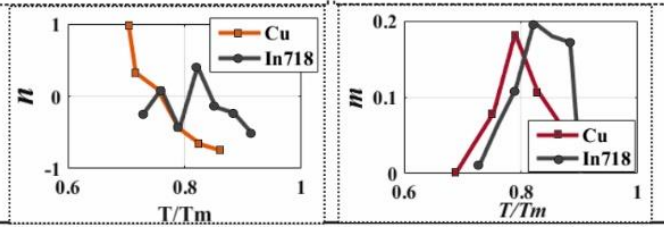

(e)

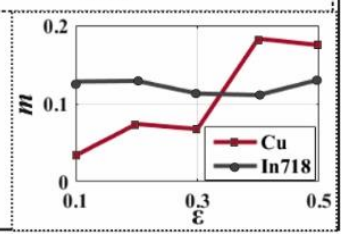

Figure 3. Illustration of the range of (a) strain, (b) strain-rate and (c) temperature typically observed in hot forging of the geometry selected, and determination of localised window wherein the material flow behaviour matches for the material pair IN718 and Cu - region of overlap of (d) strainhardening coefficient and (e) strain-rate sensitivity. 
Fig. 3 illustrates the process of material selection and detection of the "matching window" for the material couple IN718 and $\mathrm{Cu}$. Analysis of 2D flow curves to identify this window can be tedious and misleading owing to the number of curves that need to be compared, possibility from variation in dataset format, possible for coexistence of hardening and softening and so on (as can be surmised from the flow curves provided in the Appendix). Similarly, as mentioned, matching only the strain hardening $(n)$ or strain-rate sensitivity $(m)$ or thermal sensitivity $(Q)$ is not sufficient and instead a "window" in which all three are similar between the parent material and scaled material twin is required. Hence, the suggested methodology involving surface plot comparison of $n, m$ and $Q$ was employed.

It is clearly visible here that despite the fact that both IN718 and $\mathrm{Cu}$ are single phase materials and similar mechanisms of viscoplastic deformation can be expected, mechanical behaviour is very different. It is revealed on examination of Fig. 3(d and e) that a window exists but only over a restricted range of temperatures, strain rates and strains where the strain hardening $n$, same strain rate sensitivity $m$ and thermal sensitivity $Q$ match between the parent and scaled-material twin. Table 1 summarises the "matching windows" for the material couple. If it can be assumed that the majority of the points during the forging remain in the range of temperatures and strain rates that does not differ much from the "matching window", then there is a possibility to conduct scaled tests with the scaled-material twin and expect results to be not too far from the expected similitude. This assumption is experimentally investigated in Section 4.

Table 1. Matching Window - Regions of $\varepsilon, \dot{\varepsilon}, T$ - where the strain hardening $n$, same strain rate sensitivity $m$ and thermal sensitivity $Q$ match for the material twins

\begin{tabular}{|l|l|l|l|l|l|l|}
\hline \multicolumn{9}{|c|}{ Regions of Overlap } \\
\hline & \multicolumn{2}{|c|}{ Strain } & \multicolumn{2}{c|}{ Strain-rate (1/s) } & \multicolumn{2}{c|}{ Temperature K } \\
\cline { 2 - 8 } & IN718 & $\mathrm{Cu}$ & IN718 & $\mathrm{Cu}$ & IN718 & $\mathrm{Cu}$ \\
\hline Strain hardening $(n)$ & $0.35-0.5$ & $\begin{array}{l}0.35- \\
0.5\end{array}$ & $1-10$ & $1-10$ & $1223-1293$ & $1053-1113$ \\
\hline Strain-rate sensitivity $(m)$ & $0.2-0.4$ & $0.2-0.4$ & $0.1-1$ & $0.1-1$ & $1293-1353$ & $1113-1163$ \\
\hline Temperature sensitivity $(Q)$ & $0.4-0.5$ & $0.4-0.5$ & $0.1-1$ & $0.1-1$ & $1293-1323$ & $1083-1133$ \\
\hline
\end{tabular}

\section{Experimental study of feasibility of scaled modelling}

The theory in Section 2 revealed that for thermomechanical physics there exist scaled finite-similitude solutions. However, the existence of solutions is not sufficient for the creation of practical scaled experiments as discussed in Section 3 and a degree of mismatch must be anticipated for all but the most trivial cases. This section examines if the creation of process windows is sufficient to create matching model materials for sound scaled experimentation.

\subsection{Design of Scaled Experiments - Practical}


Experiments performed on three important aerospace alloys, namely IN718, Ti6A14V and AA2099, were considered as parent physical space in this work and scaled-material twins for these alloys were sought. It is known that under hot forging conditions, material deform by different mechanisms and these mechanisms dictate the material flow and the forging conditions employed. Dynamic recrystallization for example is frequently associated with flow softening, and forging temperatures and strain-rates are chosen to encourage recrystallisation. Scaling theories, including the new one proposed, cannot account for these microstructural aspects of the material. To compensate for it, as a first step, the dominant deformation mechanism for the parent materials - IN718, Ti6Al4V and AA2099 - were identified, i.e., dynamic recrystallisation, phase change, and precipitation/recrystallization, respectively. Candidate materials exhibiting similar deformation characteristics - ETP Cu, 1020 Mild Steel and AA6082, respectively - were then chosen for scaled material twins (as shown in Fig. 4). The candidate materials were chosen from a pool of materials that can be easily procured and are relatively inexpensive. The cost ratio for the material pairs were - IN718/Cu - 3; Ti6Al4V/Mild Steel - 2.5; Al2099/A16082 - 32. The flow stress data for all materials were obtained from literature (Prasad et al., 2015, Zhang et al., 2014) and interpolated/extrapolated using the Hansel-Spittel approximation (Eq. 18). The flow curves for all materials are provided in the Appendix.

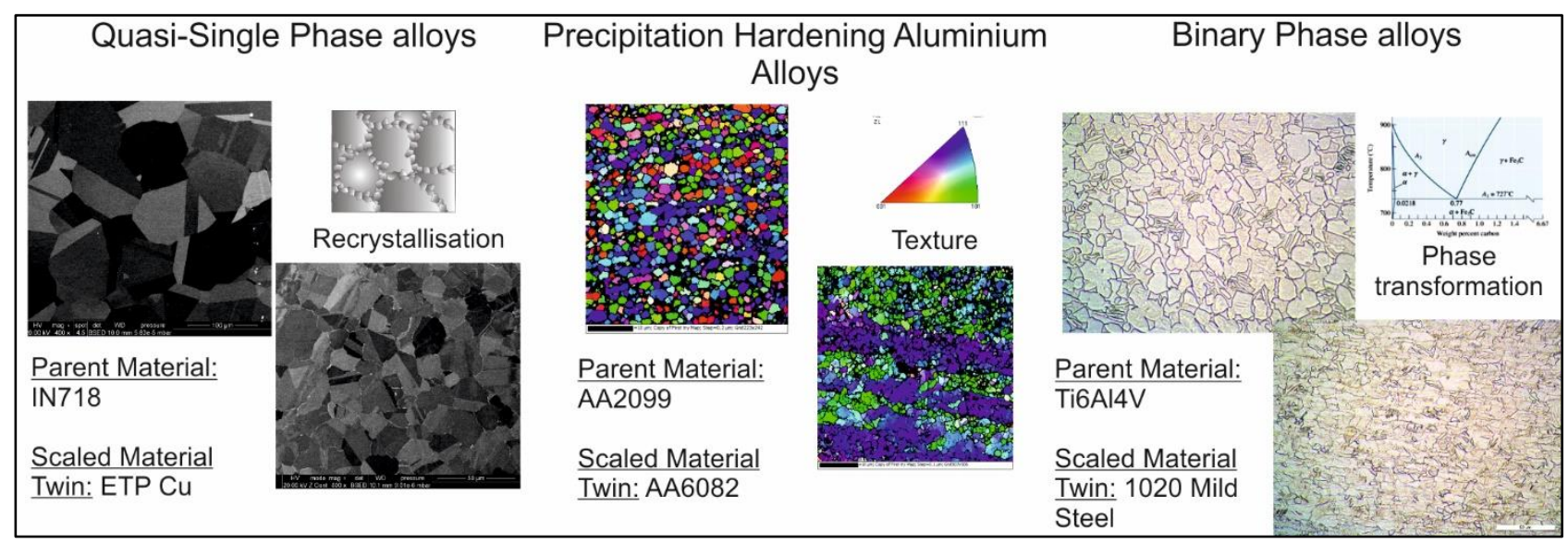

Figure 4. Summary of the relevant materials dealt with in the study and the dominant microstructural mechanism in parent materials and scaled material twins.

The geometry employed for the study is shown in Fig. 5. This particular geometry was chosen as it has different sections that require a change in the direction of material flow during forging, and forging it using a screw press results in non-uniform and non-monotonous evolution of strain, strainrate and temperature within the body through the process. Unlike laboratory-scale uniaxial tests, the stress-state is tri-axial and the triaxiality parameter $\eta$ (mean stress/equivalent stress) varies from point to point in the workpiece. The complex triaxiality state makes the part sensitive to improper forging conditions. This type of geometry offers some resemblance to those used in an industrial 
forming process, whilst remaining simple enough for a detailed analysis. The parent geometry dimensions were scaled down twice $(\beta=0.5)$ to achieve the scaled forging geometry (see Fig. 5). Starting from appropriately scaled preform cylinders, the parts were fully forged until a flash of 1 $\mathrm{mm}$ and $0.5 \mathrm{~mm}$ thickness is formed in full-scale and scaled down trials, respectively. In certain cases, the forging could not be completed in the full-scale trials due to insufficient energy, especially when forging at low temperatures close to the boundary of a stable forging temperature range. In such cases, the scaled down forging was carried out such as to ensure $\beta=0.5$ by using appropriate separation plates between the dies. Equations (7) to (12) were used to determine the scaling parameters, which in turn were used in the design of the scaled experiments (the parameters are summarised in Table 3 ). All forgings described in the work were carried out using a 2100 tonne Schuler AG screw press. The main characteristics of this press are summarised in Fig. 5. Although the press is of small scale compared to many large-scale industrial forging press, it displays all the complexities experienced in industrial-scale forging. Although the scaled trials were planned to ensure similitude, few trials resulted in underfilling, cracking, and excess flash, which were not observed in the full-scale trials. These were due to the difficulty in ensuring the exact conditions demanded for similitude and the second was the higher sensitivity of the scaled down trials to changes in forging conditions. The use of digital twins helped mitigate some of these difficulties as explained in the later sections.

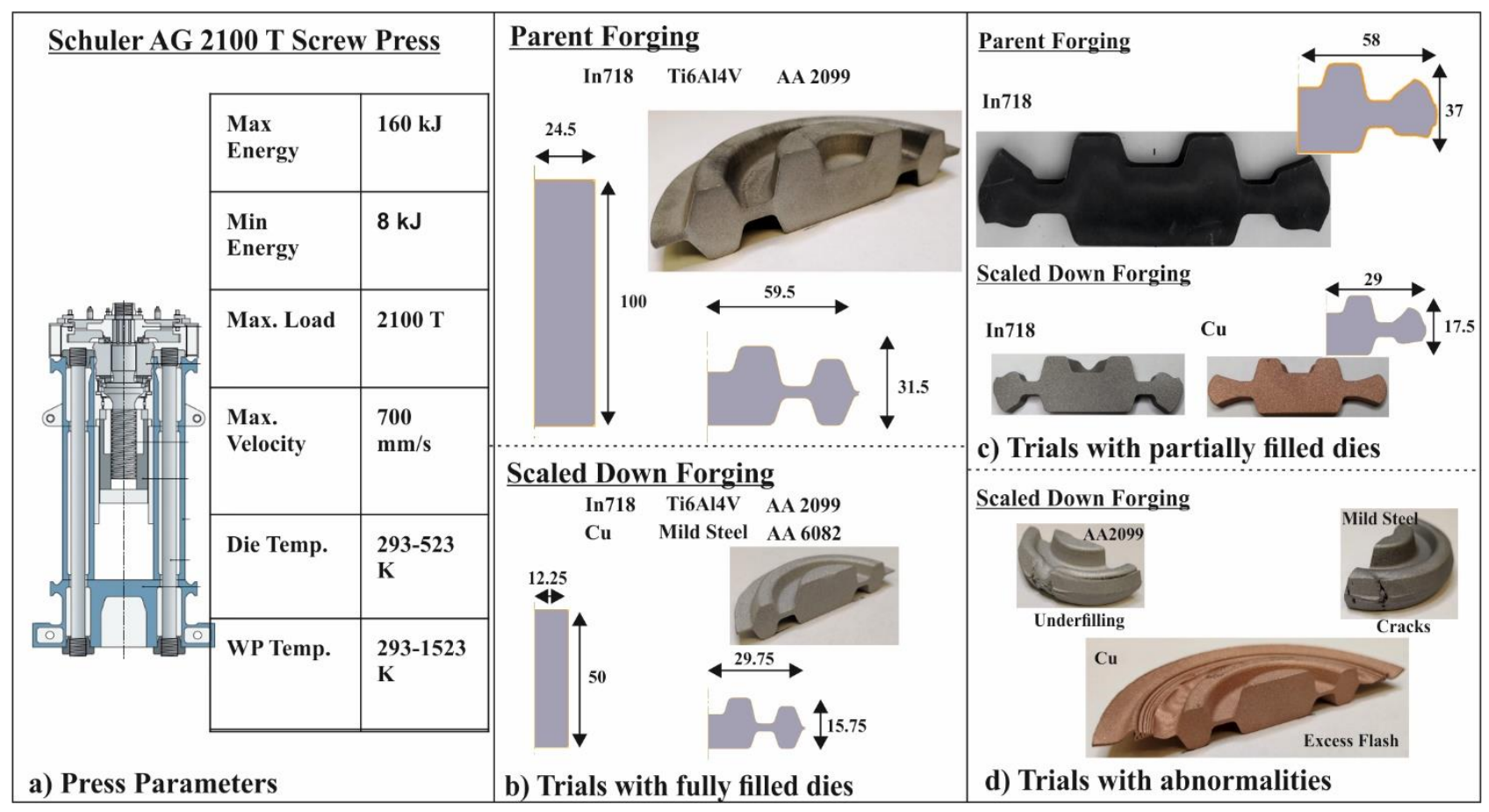

Figure 5. Characteristics of the $2100 \mathrm{~T}$ screw press and the geometry used in the forging trials.

Table 2 summarises all the experiments carried out as part of the present work. Deciding on the forging temperature is complicated as detailed in Section 3.2. For the present press, it was possible to heat the dies up to $523 \mathrm{~K}$. However, owing to the size of the die and convective heat loss, the average temperatures achieved were $453-503 \mathrm{~K}$ and the top die tended to be $20-30 \mathrm{~K}$ hotter than the 
bottom die, as can be seen from the table. The workpieces were heated in an induction furnace, with a capacity to reach up to $1523 \mathrm{~K}$. 
Table 2. Summary of all trials carried out

\begin{tabular}{|c|c|c|c|c|c|c|c|c|c|c|c|c|}
\hline \multicolumn{6}{|c|}{ Parent Forging Trials } & \multicolumn{7}{|c|}{ Scaled Down Trials } \\
\hline \multirow[b]{2}{*}{ Material } & \multirow[b]{2}{*}{$\begin{array}{c}\text { Forging } \\
\mathrm{T} \text { in } \mathrm{K}\end{array}$} & \multicolumn{2}{|c|}{ Die $T$ in $K$} & \multirow[b]{2}{*}{$\begin{array}{c}\text { Energy } \\
(\%)\end{array}$} & \multirow[b]{2}{*}{$\begin{array}{l}\text { Time } \\
(\mathrm{ms})\end{array}$} & \multirow[b]{2}{*}{ Material } & \multirow[b]{2}{*}{$\begin{array}{c}\text { Forging } \\
\mathrm{T} \text { in } \mathrm{K}\end{array}$} & \multicolumn{2}{|c|}{ Die $\mathrm{T}$ in $\mathrm{K}$} & \multirow{2}{*}{$\begin{array}{c}\text { Energy } \\
(\%)\end{array}$} & \multirow{2}{*}{$\begin{array}{c}\text { Die } \\
\text { Separator } \\
\text { distance } \\
(\mathrm{mm})\end{array}$} & \multirow[b]{2}{*}{$\begin{array}{l}\text { Time } \\
(\mathrm{ms})\end{array}$} \\
\hline & & Top & Btm & & & & & Top & Btm & & & \\
\hline \multirow{3}{*}{ IN718 } & \multirow{3}{*}{1243} & \multirow{3}{*}{498} & \multirow{3}{*}{473} & \multirow{3}{*}{75} & \multirow{3}{*}{200} & ETP-Cu & 943 & 493 & 473 & 5 & 3.5 & 240 \\
\hline & & & & & & ETP-Cu & 1053 & 493 & 473 & 5 & 5 & 238 \\
\hline & & & & & & IN718 & 1243 & 493 & 473 & 10 & - & 228 \\
\hline \multirow{3}{*}{ IN718 } & \multirow{3}{*}{1353} & \multirow{3}{*}{498} & \multirow{3}{*}{473} & \multirow{3}{*}{75} & \multirow{3}{*}{180} & ETP-Cu & 1023 & 493 & 473 & 5 & 5 & 231 \\
\hline & & & & & & ETP-Cu & 1143 & 493 & 473 & 5 & - & 245 \\
\hline & & & & & & IN718 & 1353 & 493 & 473 & 9 & - & 234 \\
\hline \multirow{3}{*}{ AA2099 } & \multirow{3}{*}{573} & \multirow{3}{*}{498} & \multirow{3}{*}{478} & \multirow{3}{*}{40} & \multirow{3}{*}{280} & AA6082 & 563 & 503 & 448 & 5 & - & 285 \\
\hline & & & & & & AA6082 & 623 & 493 & 453 & 5 & - & 270 \\
\hline & & & & & & AA2099 & 573 & 503 & 443 & 4 & 5 & 266 \\
\hline \multirow{3}{*}{ AA2099 } & & & & & & AA6082 & 673 & 493 & 453 & 5 & - & 265 \\
\hline & 623 & 503 & 483 & 35 & 260 & AA6082 & 723 & 493 & 453 & 5 & - & 265 \\
\hline & & & & & & AA2099 & 623 & 503 & 463 & 5 & - & 308 \\
\hline & & & & & & MS & 1053 & 503 & 463 & 5 & - & 270 \\
\hline Ti6Al4V & 900 & 493 & 473 & 50 & 220 & MS & 1103 & 503 & 463 & 5 & - & 275 \\
\hline & & & & & & Ti6Al4V & 1173 & 493 & 453 & 7 & - & 238 \\
\hline & & & & & & MS & 1123 & 503 & 463 & 5 & - & 300 \\
\hline Ti6Al4V & 950 & 493 & 473 & 50 & 200 & MS & 1163 & 503 & 463 & 5 & - & 305 \\
\hline & & & & & & Ti6Al4V & 1223 & 493 & 453 & 6 & - & 245 \\
\hline
\end{tabular}


The choice of forging temperature for each parent and modelling material couple were decided based on the scaling parameter $\xi^{T}$. The choice of the reference temperature for scaling is nontrivial as discussed and attempts were made in this study to understand the effect of this choice, which is discussed in detail in the next section.

After forging, the formed part was cleaned of scale and lubricant, sliced along the middle, measured, polished and also studied under the microscope. Logged data - Load, Position and Velocity - from the screw press were retrieved and processed. Surface and real-time die temperature measurements were also recorded. This data were used to calibrate FE models and generate digital twins as discussed in Section 3.1.2 (Fig. 2). All FE models used in the present work were developed using the commercial metal forming software QForm.

\subsection{Experiments with Scaled Material Twins}

Scaled experiments with scaled material twins were carried out to study the applicability of the exact scaling theory. Following the logic outlined in Section 3.2, first the scaling factor $\beta$ was chosen based on the capacity of the forging equipment. When carrying out scaled trials with $\beta=0.5$, there was a choice to opt for either the same strain-rate or velocity, but never both. The strain-rates and velocities in the physical and trial space can be related by the equations $\dot{\varepsilon}_{t s}=g \dot{\varepsilon}_{p s}$ and $v_{p s}=g \beta^{-1} v_{t s}$, respectively. The time scaling parameter $g(g=1)$ was chosen to ensure identical strain rates in both full-scale and half-scale models. However, achieving $g=1$ was difficult in practice with the only means of control being the screw press energy. Through means of preliminary simulations the same time of forging as the parent process was achieved, and the energy value found from the preliminary simulations were then used in the forgings trials. There was a degree of uncertainty in the energy delivered (around 10\% error) or in some cases the energy required was below the minimum energy limit of the press, which is $5 \%$. In such cases, calibrated FE models were used to construct digital twins and these were subsequently employed for analysis purposes. On specifying $\beta$ and $g$, and on the choice of the scaled-material twin, $\alpha^{\rho}, \xi^{\sigma}$ and $\xi^{T}$ are determined to achieve a narrow window of flow overlap. These values provide the dependent scaling parameters, i.e. $\alpha^{v}=g \beta^{-1} \alpha^{\rho}$, $\alpha^{u}=\beta^{-1} \alpha^{\rho}$ and $\alpha^{e}=g^{2} \beta^{-2} \alpha^{\rho}$. The scaling parameters for the different material couples are summarised in Table 3.

Table 3. Important dependent and independent scaling parameters

\begin{tabular}{|c|c|c|c|c|c|}
\hline $\begin{array}{c}\beta \\
\text { (Length) }\end{array}$ & $\begin{array}{c}g \\
\text { (Time) }\end{array}$ & $\alpha^{\rho}$ (Density) & $\xi^{\sigma}$ (Stress) & $\begin{array}{c}\xi^{T} \\
\text { (Temperature) }\end{array}$ & $\alpha^{e}$ (Energy) \\
\hline \multicolumn{7}{|c|}{ IN718 and ETP-Cu pair } \\
\hline 0.5 & 1 & 0.14 & 0.6 & 0.84 & 0.04 \\
\hline
\end{tabular}




\begin{tabular}{|l|l|c|c|c|c|}
\hline \multicolumn{7}{|c|}{ Ti6A14V and Mild Steel pair } \\
\hline 0.5 & 1 & 0.21 & 0.45 & 0.87 & 0.05 \\
\hline \multicolumn{7}{|c|}{ AA2099 and AA6082 pair } \\
\hline 0.5 & 1 & 0.13 & 0.5 & 0.90 & 0.03 \\
\hline
\end{tabular}

With the calculated scaling parameters, the conditions for the scaled forgings were decided, as summarised in Table 2. Recall that for reference temperature either the melting temperature or the characteristic temperature of the alloys can be used. The characteristic temperature is based on the dominant deformation mechanism of the alloy - recrystallization ( $T^{\mathrm{rec}}$ for $\mathrm{IN} 718 / \mathrm{Cu}$ ), phase transformations ( $T^{\mathrm{pt}}$ for Ti6Al4V/Mild steel), precipitation ( $T^{\mathrm{ps}}$ for AA2099/AA6082). However, in some cases, this can provide temperatures outside the stable forging window for the scaled down process or in a few cases outside the window of rheology matching. Table 4 below summarises the temperatures under consideration for the material couples and temperature scaling parameter $\xi^{T}$ for each of them. As can be readily seen, the value of $\xi^{T}$ depends strongly on the choice of reference temperature and the choice is not straightforward.

Table 4. Critical temperatures for determination of $\xi^{T}$

\begin{tabular}{|c|c|c|c|c|c|c|c|c|c|}
\hline $\mathbf{T}$ in $\mathbf{K}$ & IN718 & $\mathbf{C u}$ & $\xi^{T}$ & Ti6Al4V & MS & $\xi^{T}$ & AA2099 & $\begin{array}{c}\text { AA608 } \\
2\end{array}$ & $\xi^{T}$ \\
\hline Melting & 609 & 358 & 0.84 & 1873 & 1783 & 0.95 & 923 & 828 & 0.89 \\
\hline $\begin{array}{l}\text { Characte } \\
\text { ristic }\end{array}$ & 1223 & 923 & 0.75 & 1253 & 1103 & 0.88 & $493-593$ & $\begin{array}{c}473- \\
578\end{array}$ & 0.96 \\
\hline $\begin{array}{c}\text { Stable } \\
\text { Forging } \\
\text { range }\end{array}$ & $\begin{array}{c}1173- \\
1323\end{array}$ & $\begin{array}{c}1023- \\
1173\end{array}$ & $\begin{array}{c}0.87- \\
0.89\end{array}$ & $\begin{array}{c}1023- \\
1223\end{array}$ & $\begin{array}{l}1173- \\
1473\end{array}$ & $\begin{array}{c}1.14- \\
1.20\end{array}$ & $693-773$ & $\begin{array}{c}593- \\
633 / 73 \\
3-803 \\
\end{array}$ & $\begin{array}{c}0.86- \\
0.99\end{array}$ \\
\hline $\begin{array}{c}\text { Window } \\
\text { of } \\
\text { overlap }\end{array}$ & $\begin{array}{l}1223- \\
1293\end{array}$ & $\begin{array}{c}1053- \\
1113\end{array}$ & $\begin{array}{c}0.82- \\
0.86\end{array}$ & $\begin{array}{c}1223- \\
1313\end{array}$ & $\begin{array}{l}1113- \\
1203\end{array}$ & $\begin{array}{c}0.91- \\
0.92\end{array}$ & $683-763$ & $\begin{array}{l}623- \\
703\end{array}$ & $\begin{array}{l}0.91- \\
0.92\end{array}$ \\
\hline
\end{tabular}

To study the significance of the choice of the reference temperature, forgings with scaled material twins were carried out using both the melting temperature and the characteristic temperature as reference. This choice is critical as is illustrated by the example of a mild steel (MS) forgings as shown in Fig. 6. Using the phase-change temperature as the reference, it was possible to forge MS without any defects and close to the parent Ti6Al4V trials (Fig. 6(d)), while the use of melting point as the reference lead to cracks as it brought the material too close to the transition temperature, and thereby instability (Fig. 6(e)). This can be used as a guidance measure for deciding the reference temperature. The characteristic temperature may vary depending on the alloy, but can provide a means to determine stable forging zones in the scaled material twins. 


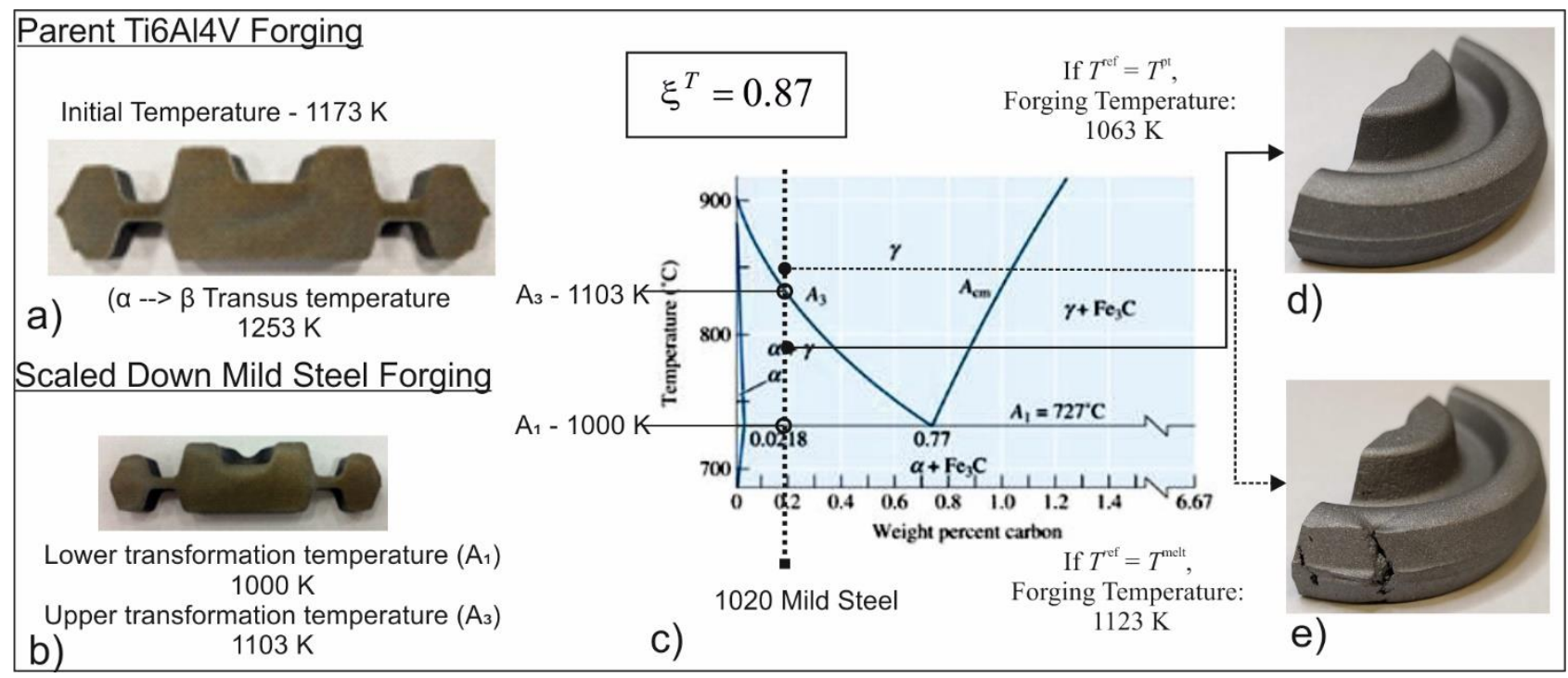

Figure 6. Comparison of the parent (Ti6Al4V) and scaled down (Mild Steel) forging with emphasis on the importance of the choice of reference temperature: a) Parent forging of Ti6Al4V with the forging temperature and the characteristic temperature (phase transformation, $T_{p s}^{p t}$ ); b) scaled down forging of mild steel with the characteristic temperatures $\left.\left(T_{t s}^{p t}\right) ; \mathrm{c}\right)$ phase diagram of 1020 mild steel overlaid with the different temperatures considered; and forged geometries resulting from the use of d) characteristic temperature $\left(T^{\mathrm{pt}}\right)$ and e) melting temperature $\left(T^{\text {melt }}\right)$ as the reference temperature for temperature scaling $\left(\xi^{T}\right)$

Moreover, fixing the forging temperature for the scaled down process, solely means fixing the initial temperature of the billet. Unfortunately, realistically, the ambient temperature and die temperatures cannot similarly be made proportional, and this leads to disproportionate convective heat transfer. Furthermore, matching the materials for similar rheological behaviour does not ensure similar thermal behaviour. For instance, while copper exhibits similar microstructural and rheological behaviour ( $n$ , $m$ and $Q$ ) as does IN718 in the narrow window selected, it has a markedly different thermal conductivity, as can be seen from Table 5. This means that although the initial preform temperature is chosen based on $\xi^{T}$, the temperature distribution in the workpiece during the actual forging will be drastically different in the parent and scaled-material twin (as seen from Fig, 7(b)), and so will in turn the material flow (as shown in Fig. 7(a)). Figure 7(c) shows the temperature profile recorded for three closely located points in digital twins generated for IN718 and $\mathrm{Cu}$. As is evident, the temperature increases notably in IN718 and this increase is different for different points, as opposed to the uniform near negligible increase observed in $\mathrm{Cu}$. Many notable works on physical modelling in the past have discussed this difficulty in achieving thermal similarity (Altan et al., 1970, Pawelski, 1992). 


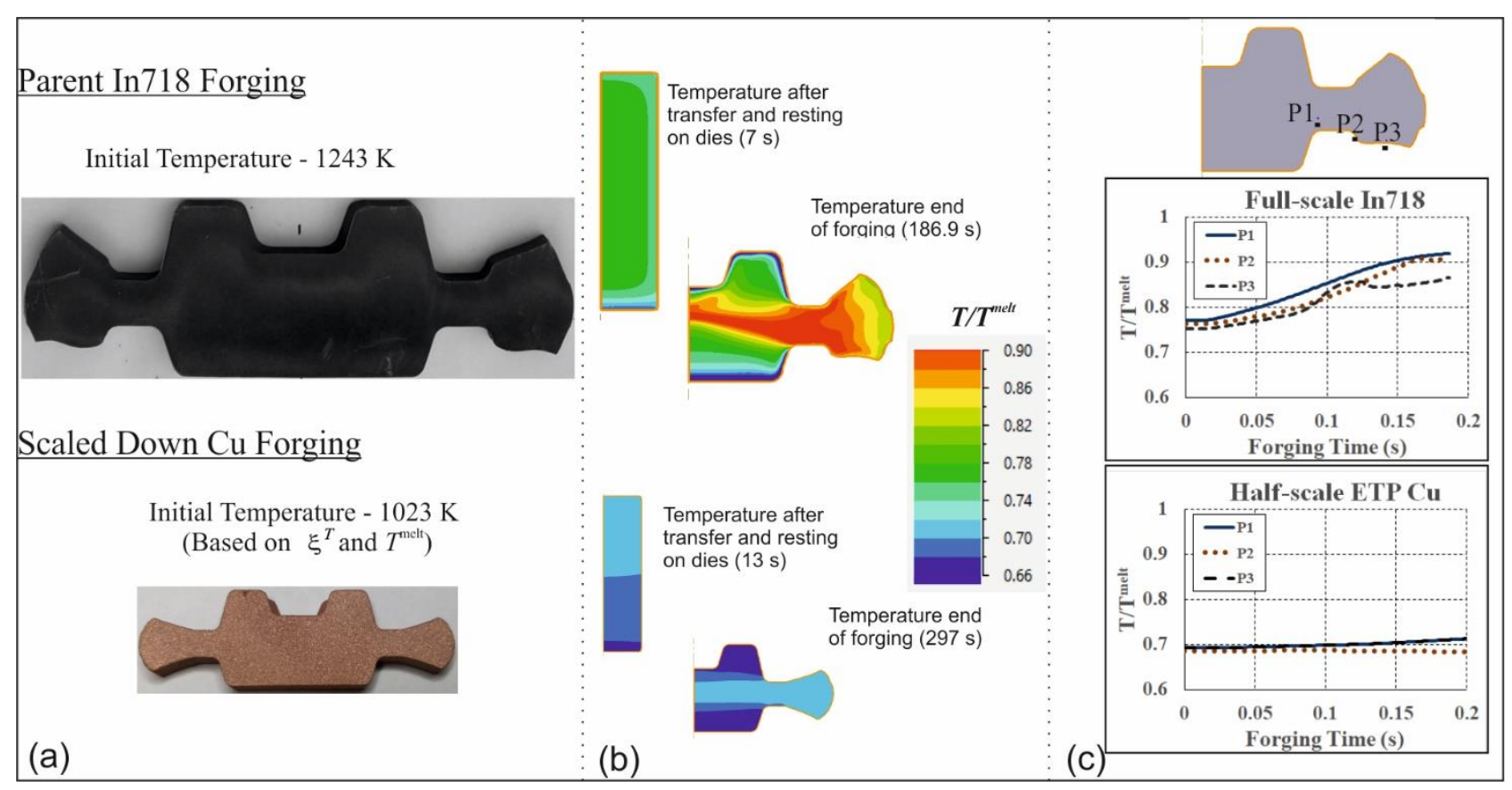

Figure 7. Comparison of the parent (IN718) and scaled down (ETP-Cu) forging with emphasis on the thermal behaviour of materials

Table 5. Thermal properties for testing materials.

\begin{tabular}{|c|c|c|c|}
\hline Material & $\begin{array}{c}\text { Specific Heat } \\
\mathrm{J} /(\mathrm{kgK})\end{array}$ & $\begin{array}{c}\text { Thermal } \\
\text { Conductivity } \\
\mathrm{W} /(\mathrm{mK})\end{array}$ & $\begin{array}{c}\text { Convective heat } \\
\text { transfer coefficient } \\
\mathrm{W} /\left(\mathrm{m}^{2} \mathrm{~K}\right)\end{array}$ \\
\hline IN718 & 435 & 22 & 22500 \\
\hline $\mathrm{Cu}$ & 390 & 410 & 45000 \\
\hline Ti6A14V & 930 & 17.4 & 45000 \\
\hline Mild Steel & 660 & 26.9 & 30000 \\
\hline AA2099 & 1203 & 88 & 40000 \\
\hline AA6082 & 935 & 174 & 40000 \\
\hline
\end{tabular}

Given the difficulty in carrying out exact scaled trials and the all-so-very-apparent complications in using different materials, it can be argued if there is any sense in carrying out scaled trials using scaled-material twins. However, as is witnessed by the number of successful physical modelling trials in the past, such scaled trials can be useful if used for specific purposes. More importantly, one of the reasons for the decline in physical modelling is that even though the differences can be apparent in cases (such as the cracks and different geometry), the reason for the difference is not always readily apparent or quantifiable. The use of physical and numerical modelling (using digital twins) offers a unique capability to understand these differences more clearly and quantify them.

The local and global measure of similitude formulated in Section 3.1 provides an ability to quantify, visualise and analyse the difference in behaviour of material couples. Table 6 summarises the local and global measures of similarity for the material couples. An example of the methodology used to calculate the measures of similitude is provided in Figure 8 for IN718. Several points in the FE digital 
twin of the parent forging were tracked through the process. Similarly, points with a one-to-one scaled correspondence in the FE digital twin of the scaled process were tracked through all the time steps. Following this, Eq. 16a and 16b were employed to find local measures of similitude. These measures are plotted against the time step for the digital twins in Fig. 8(a). The spatial distribution of these points and the overall geometry obtained at different key time steps are represented in Fig. 8(b). For clarity, only 5 points are provided in this example, while in reality 200 points (20 rows, 10 columns) were used for calculating the values provided in Table 6. To obtain the global measure of similarity, the absolute difference between the coordinates were normalised using either the displacement of the corresponding point through the forging $(\mathrm{del})$ or using the tool stroke during forging as reference $\left(\right.$ del $\left._{0}\right)$. The tool stroke was used as the normalising length here to account for the varying degrees of completion of the full-scale process, which were then used to stop the scaled down process accordingly.

Two key observations that can be made from the analysis are as follows: for all material couples analysed, there is a good level of similitude between the parent and modelling material in zones with limited or uniaxial deformation $\left(\Delta_{2}<5\right.$ in Points P1 and P2 in Fig. 8). The difference $\Delta_{2}$ is large in zones involving direction change and large deformation (Point P3 in the web regions of the disc as shown in Fig. 8). Secondly, the local similarity is difficult to achieve in material couples where the differences in thermal properties are quite large (the case of IN718 and Cu). In cases involving material couples with similar thermal properties, the local similarity can be improved by choosing the reference temperature for scaling properly, as can be derived from the $\Delta_{2}$ values summarised for different scenarios in Table 6.

Table 6. Measure of similarity between digital twins of forging with parent material and scaled material twins

\begin{tabular}{|c|c|c|c|c|}
\hline $\begin{array}{l}\text { Material } \\
\text { Pair }\end{array}$ & $\begin{array}{c}\text { Reference } \\
\text { temperature }\end{array}$ & $\begin{array}{c}\ell_{0} \\
\text { Characteristic } \\
\text { normalizing } \\
\text { length (Tool } \\
\text { Stroke mm) } \\
\end{array}$ & $\begin{array}{c}\text { Max_Absolute } \\
\text { Difference in mm } \\
\max _{\substack{\boldsymbol{x}_{0 s}^{m} \in \Omega_{0 p s}^{m} \\
t_{p s} \in\left[0, t_{p s}^{*}\right]}}\left\|\boldsymbol{x}_{p s}^{m}-\beta^{-1} \boldsymbol{x}_{t s}^{m}\right\|_{2} \\
\end{array}$ & $\begin{array}{c}\text { Global measure of } \\
\operatorname{similarity}\left(\Delta_{2}\right) \text { in \% } \\
\Delta_{2}=\max _{\boldsymbol{x}_{0 p s}^{m} \in \Omega_{0 p s}^{m}} \delta\left(\boldsymbol{x}_{0 p s}^{m}, t_{p s}^{*}\right)\end{array}$ \\
\hline \multirow{2}{*}{$\begin{array}{l}\text { IN718 - } \\
\text { ETP Cu }\end{array}$} & Melting & 73 & 8.2 & 11.3 \\
\hline & Recrystallisation & 73 & 8.6 & 11.7 \\
\hline \multirow{2}{*}{$\begin{array}{l}\text { Ti6Al4V - } \\
\text { Mild Steel }\end{array}$} & Melting & 78.5 & 8.5 & 10.9 \\
\hline & $\begin{array}{c}\text { Phase } \\
\text { Transformation }\end{array}$ & 78.5 & 7.3 & 9.3 \\
\hline \multirow{2}{*}{$\begin{array}{l}\text { AA2099 - } \\
\text { AA6082 }\end{array}$} & Melting & 78 & 5.4 & 6.9 \\
\hline & Precipitation & 78 & 5.6 & 7.2 \\
\hline
\end{tabular}




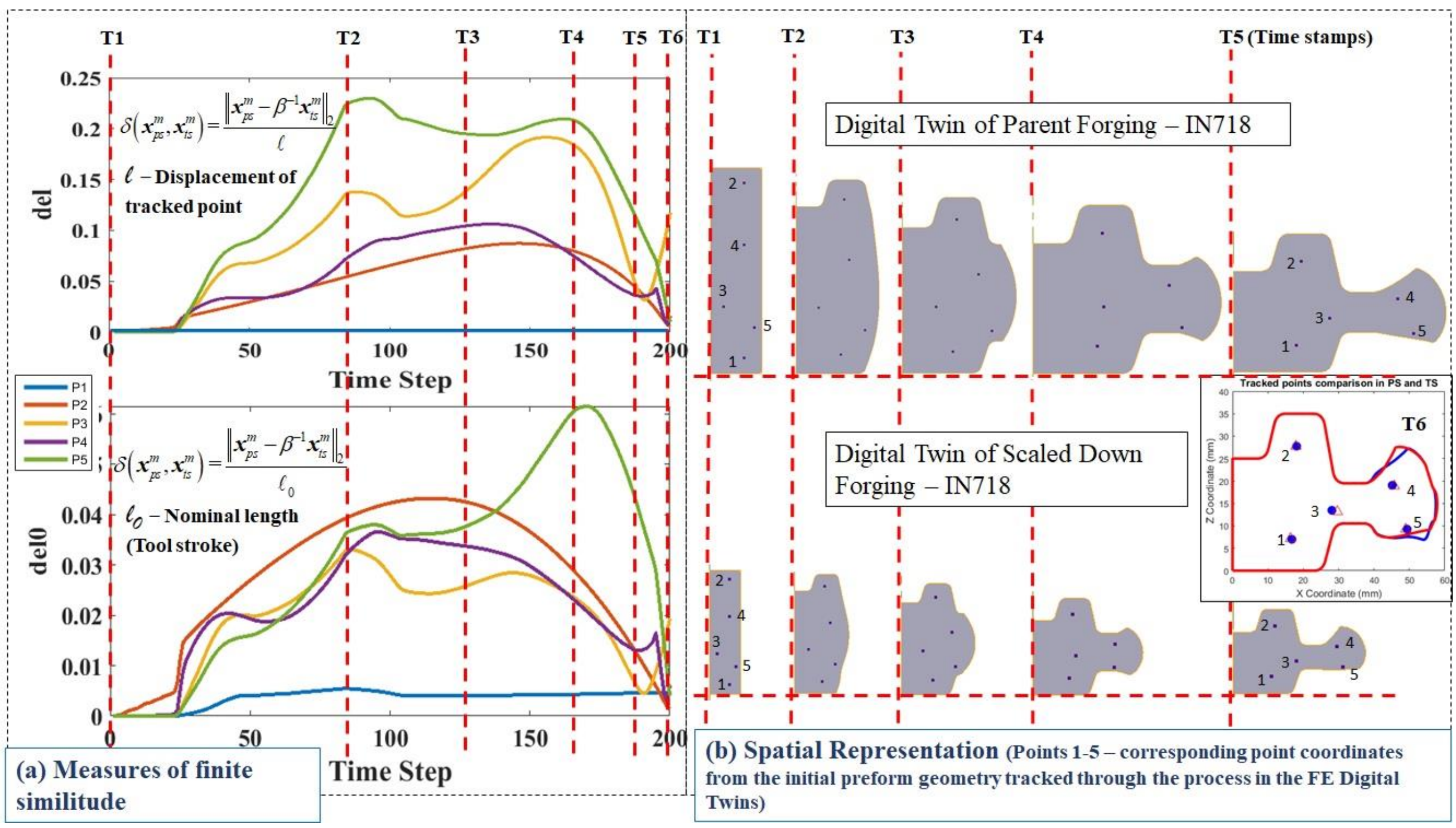

Figure 8. An example of the methodology used to calculate the measures of similitude for IN718: (a) measures of similitude calculated from digital twins using Eq. 16a and Eq. 16b plotted against the time step (b) Spatial representation of key points in the digital twins of parent and scaled down forgings tracked through the time steps 
In some cases, for example in the case of Ti6Al4V forgings, the use of same material in scaled trials can yield better results, as discussed in the next section. In other cases, such as the case of AA2099 and AA6082, the modelling materials come very close, and sometimes better than using the same material. In this case, the flow behaviour can be closely matched as can be witnessed in the flow lines in Fig. 9. Given that the cost ratio for AA2099/AA6082 is 32, it can be hugely beneficial to carry out scaled down trials using AA6082 to predict the outcomes of large-scale AA2099 forgings.

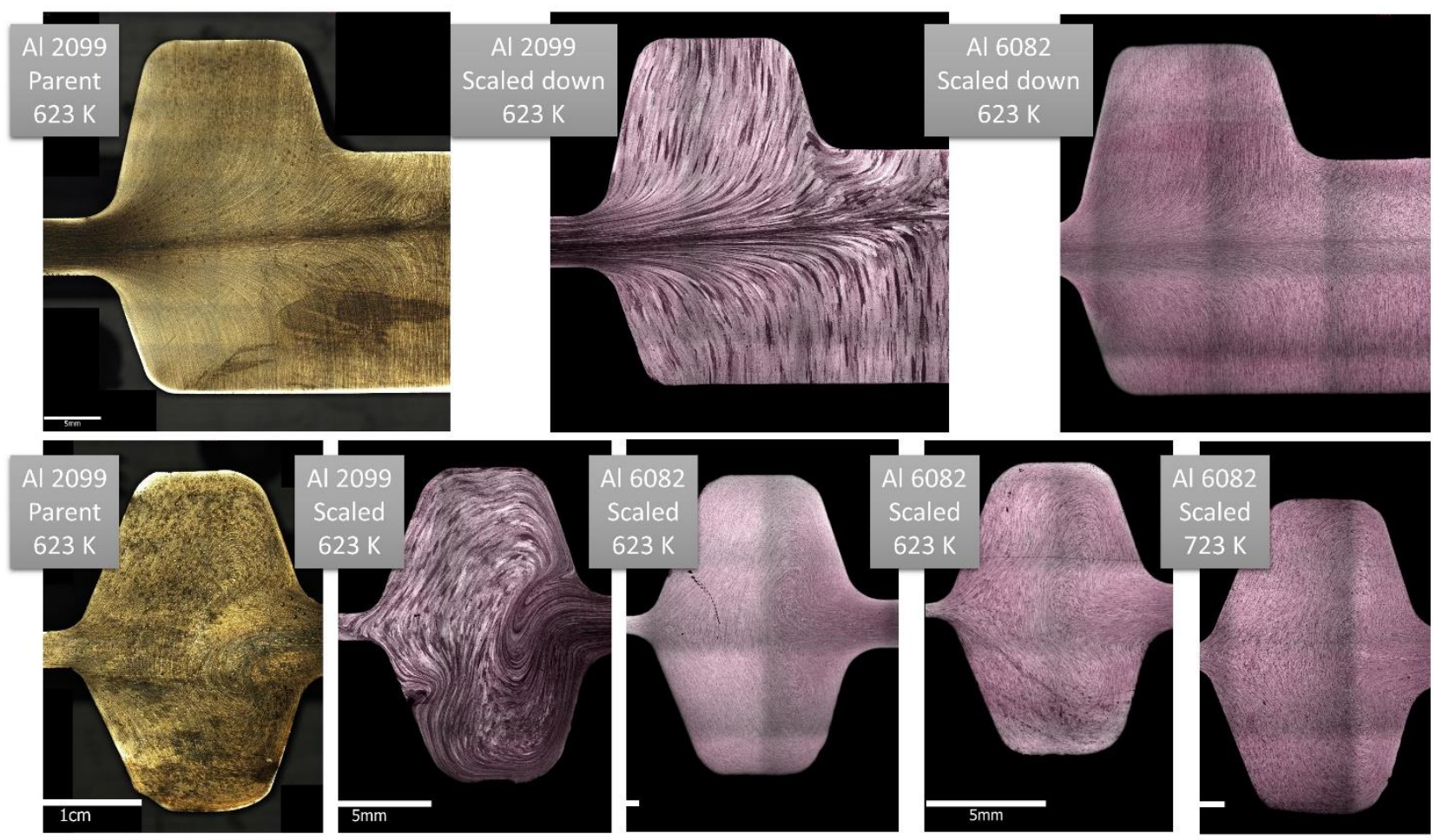

Figure 9. Comparison of the flow lines generated in full and scaled down forgings of AA2099 and AA6082 material pair.

\subsection{Scaled Down Experiments with Same Material - Inexact Scaling}

Scaled down experiments conducted with the same material as the parent forging process are of special interest. Despite the well-known fact that proportional geometrical scaling changes the behaviour of the mechanical system (Sedov, 1993, Barenblatt, 2003), miniaturised trials are very popular and often unavoidable in the metal forming industry. This increases the importance of a detailed analysis of such experiments for the sake of understanding the nature of any mismatch that arises. In this case, some of the scaling relationships derived in Section 2 will be automatically broken, so henceforth, these experiments are called "inexact scaling".

From the one hand, using the same material in the parent and scaled down experiments automatically resolves the main problem of scaled-material twins, i.e. achieving similarity in mechanical behaviour. In inexact scaling experiments, material follows the same constitutive law, so a number of the scaling parameters automatically becomes equal to 1 , e.g. $\xi^{\sigma}=1, \xi^{T}=1$ and $g=1$. This means that the 
scaled down trials must be conducted at the same temperature and have the same duration as the parent one. This does not create any problems if the process is isothermal - this is why material testing, e.g. isothermal tensile test, can be performed on similar specimens of different sizes (ASTM International, 2016, ASTM International, 2017). Unfortunately, the majority of hot forging processes are not isothermal and cooling of the workpiece due to heat exchange with the dies and air as well as adiabatic heating affects the metal flow significantly. The transport equation for energy Eq. (11) reduces (with $\alpha^{\rho}=\beta^{-3}$ and $\alpha^{e}=\beta^{-5}$ ) to

$$
\begin{gathered}
\alpha^{e} \mathbf{T}^{e}(\beta)=\frac{D^{*}}{D^{*} t_{p s}} \int_{\Omega_{p s}^{*}}\left[\rho_{p s}\right]\left(\beta^{-2}\right) u_{t s}+\left[\rho_{p s}\right] \frac{v_{p s}^{2}}{2} d V_{p s}^{*} \\
+\int_{\Gamma_{p s}^{*}}\left[\rho_{p s}\right]\left(\beta^{-2}\right) u_{t s}\left(\underline{v}_{p s}-\underline{v}_{p s}^{*}\right) \cdot \underline{n}_{p s} d \Gamma_{p s}^{*}+\int_{\Gamma_{p s}^{*}}\left[\rho_{p s}\right] \frac{v_{p s}^{2}}{2}\left(\underline{v}_{p s}-\underline{v}_{p s}^{*}\right) \cdot \underline{n}_{p s} d \Gamma_{p s}^{*} \\
+\int_{\Gamma_{p s}^{*}}\left(\beta^{-3} \underline{q}_{t s}\right) \cdot \underline{n}_{p s} d \Gamma_{p s}^{*}-\int_{\Gamma_{p s}^{*}}\left(\underline{v}_{p s} \cdot \beta^{-2} \underline{\sigma}_{t s}\right) \cdot \underline{n}_{p s} d \Gamma_{p s}^{*}=0
\end{gathered}
$$

which highlights the lack of finite similitude, since $d\left(\alpha^{e} \mathbf{T}^{e}\right) / d \beta \equiv 0$ requires that $u_{p s}=\beta^{-2} u_{t s}$, $\underline{q}_{p s}=\beta^{-3} \underline{q}_{t s}$ and $\underline{\underline{\sigma}}_{t s}=\beta^{-2} \underline{\underline{\sigma}}_{t s}$ but with identical materials $u_{p s}=u_{t s}, \underline{q}_{p s}=\beta \underline{q}_{t s}$ and $\underline{\underline{\sigma}}_{t s}=\underline{\underline{\sigma}}_{t s}$.

From a physical point of view this will manifest as a difference in the temperature distribution since the energy stored and energy released change with $\beta$ and therefore depart significantly in the case of ever smaller values of $\beta$.

As mentioned above, the three materials selected for inexact scaling tests represent three different types of materials (see Fig. 4). Owing to the difference in metallurgical nature and the dominating microstructural mechanisms, they have different specific heat capacity, different heat conductivity and different mechanical sensitivity to the temperature and strain rate, as can be seen from Table 5 . As discussed in the previous section, in regions where the differences in temperature and strain-rate trajectory are similar between the parent and scaled down experiments, the difference is not very apparent. But the main differences manifests in regions where the trajectories deviate significantly. Table 7 below summarises the differences observed in inexact scale trials for the three materials using the global measure of similarity and the follow sections briefly expounds the interesting results observed in the three materials.

Table 7. Measure of similarity between digital twins of scaled forging with same material

\begin{tabular}{|c|c|c|c|}
\hline Material Pair & $\begin{array}{c}\ell_{0} \text { Characteristic } \\
\text { normalizing length } \\
\text { (Tool Stroke mm) }\end{array}$ & $\begin{array}{c}\text { Max absolute } \\
\text { difference in mm }\end{array}$ & $\begin{array}{c}\text { Global measure of } \\
\text { similarity }\left(\Delta_{2}\right) \text { in \% }\end{array}$ \\
$\Delta_{2}=\max _{\boldsymbol{x}_{0 p s}^{m} \in \Omega_{0 p s}^{m}} \delta\left(\boldsymbol{x}_{0 p s}^{m}, t_{p s}^{*}\right)$ \\
\hline
\end{tabular}




\begin{tabular}{|c|c|c|c|}
\hline & & $\max _{\substack{\boldsymbol{x}_{0 p s}^{m} \in \Omega_{0 p s}^{m} \\
t_{p s} \in\left[0, t_{p s}^{t}\right.}}\left\|\boldsymbol{x}_{p s}^{m}-\beta^{-1} \boldsymbol{x}_{t s}^{m}\right\|_{2}$ & \\
\hline IN718 & 73 & 7.1 & 9.8 \\
\hline Ti6A14V & 78.5 & 3.1 & 3.9 \\
\hline AA2099 & 78 & 5.3 & 6.8 \\
\hline
\end{tabular}

Some of the consequences of the difference in thermomechanical histories were directly observed during forging. If the forging trials were performed under conditions corresponding to the window of stable material properties (Zhang et al., 2014) the differences in the forging may not be visible at all. However, if the forging was done under conditions closer to the boundaries of the process window, the differences can be huge. Fig. 10(a), shows two scaled down forgings with AA2099, performed at $573 \mathrm{~K}$ and $623 \mathrm{~K} .573 \mathrm{~K}$ is too low a temperature for forging AA2099, so additional cooling on contact with the dies and air can reduce the ductility and lead to the fracture, while the full-scale disks (parent) were forged without defects. Fig 10(b) shows that at $623 \mathrm{~K}$ the problem of surface fracture reduces, and it vanishes at higher temperatures.

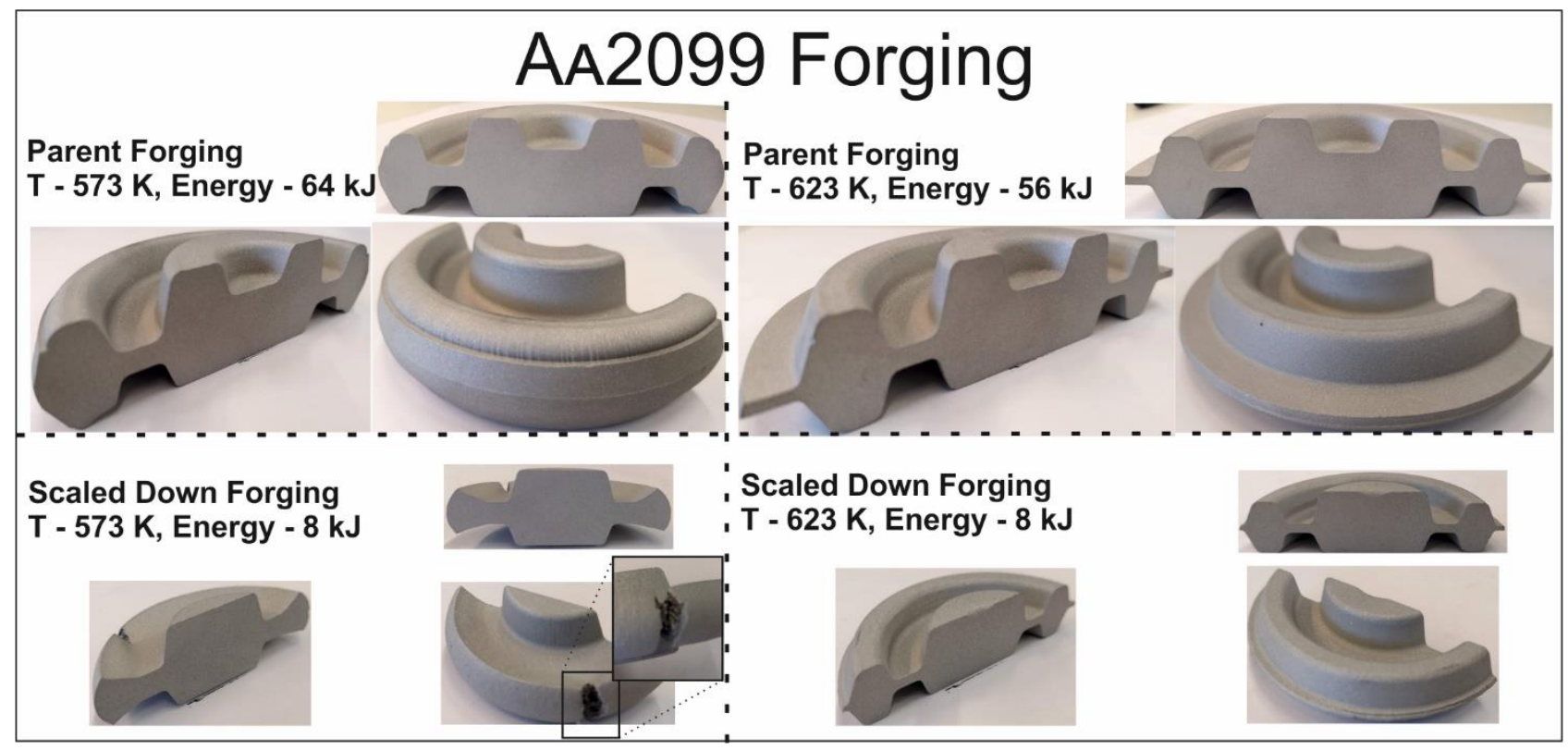

Figure 10. Illustration of the effect of temperature on aluminium forgings.

Strangely, the opposite effect were observed in forging IN718 - scaled down forgings were without defects, while the parent ones demonstrated cracks in all ranges of temperatures explored; hown in Figure 11. 
Forging of IN718

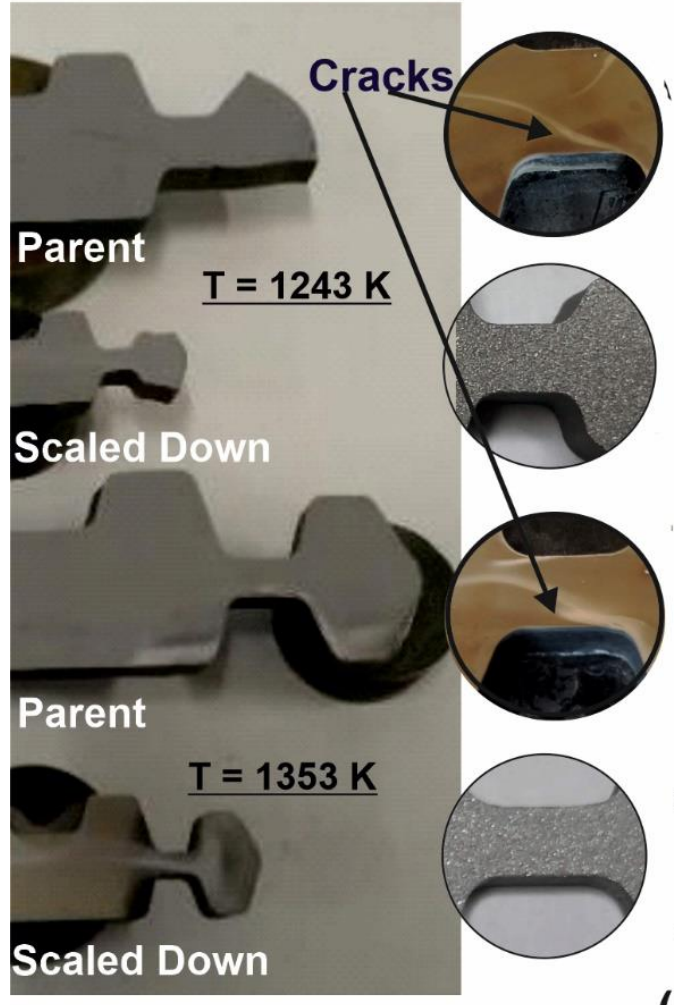

(a)

\section{Analysis of Digital twins}
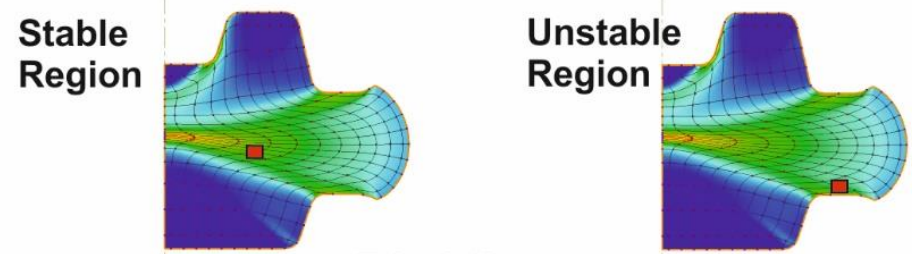

Triaxiality

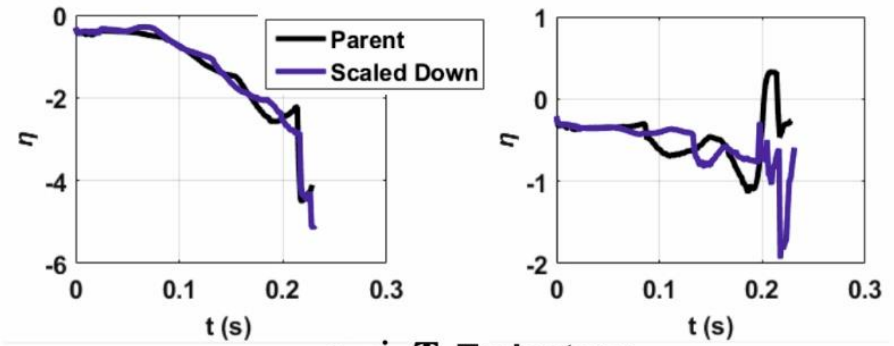

$\varepsilon, \dot{\varepsilon}, \boldsymbol{T}$ Trajectory

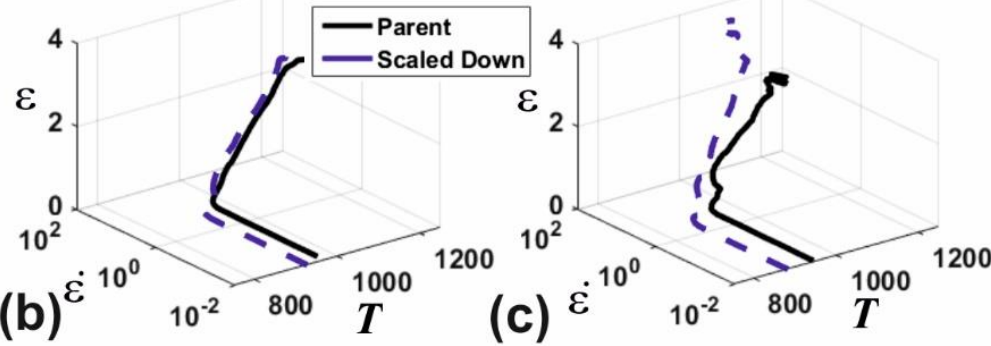

Figure 11. Comparison of the results from inexact scaling experiments with IN718 at $1243 \mathrm{~K}$ and $1353 \mathrm{~K}$ - comparison of (a) output geometry with the inset showing the cracks developed in the parent forging and triaxiality and strain, strain-rate and temperature trajectory for a point in (b) stable region and (c) unstable region

One of the possible explanations for this phenomenon is the possibility of material overheating. With the high specific heat capacity of In718, the adiabatic heating was found to be significant in FE simulations. However, in this study, the forgings were done at two temperatures $1243 \mathrm{~K}$ and $1353 \mathrm{~K}$ (see Fig. 11) and in both cases, there were no cracks in the scaled down disks. There is no direct answer to this problem from the FE simulations. However, analysis of the trajectory and triaxiality in the points sheds some light on the location and instance of the crack formation. As shown in Fig. 11 (b) and (c), in regions undergoing stable deformation, the trajectory and the triaxiality seem to be similar. On the other hand, in regions with unstable deformation, the triaxiality varied significantly and so does the trajectory. This can be used as a useful tool to identify regions of unstable deformation while carrying out scaled experimentation. Notable differences in triaxiality and trajectories between digital twins of scaled experiment and projected parent forging can be an indicator for possible failure.

This is an important area of interest for the forging industry. Flow localisation or formation of dead zones can be the reason for various macro- and micro-defects, such as folds, under-fillings, microand macro-cracks, unwanted textures and material properties. Due to this reason, it is obviously interesting to see whether scaled down trials with the actual or scaled-material twins can predict the 
stability of metal flow in large forgings. Some results of this analysis were already presented and discussed above for aluminium alloys (see Fig. 10). More general comparison for the three main materials (AA 2099, Ti6Al4V and Inconel 718) is shown in Fig. 12. A common trend observed in this series of tests is the general display of lower stability of metal flow in the scaled down trials. Scaled down forgings of AA2099 and Ti6A14V (Fig. 12(a) and 12(b)) demonstrate surface folds and complex grain-flow lines, which are not present in the parent forgings. Fig. 12(c). shows the images of two scaled down disks forged under the same forging conditions one after another. These forgings were deliberately done with the amount of screw press energy insufficient for the complete filling of the dies to imitate the conditions of the parent forging, which was incompletely forged due to low temperature and limited capacity of the press. These tests indicate how materials flow into the webs. It can be seen that the intermediate shape of the formed webs are very different - sometimes the material flows towards the top; sometimes, bottom; and sometimes, straight (grain-flow lines in IN718 and other materials also confirm this). The utilisation of digital twins of the process provides a possible explanation for this - small variations in the boundary conditions (friction or temperature of the dies). Similar variations in the boundary conditions do not produce such drastic effects in the parent forgings. This result suggests that the higher surface-to-volume ratio associated with the forging of scaled down parts can be more sensitive to boundary conditions. This suggests an idea for possible utilisation of scaled down trials for the investigation of the sensitivity of metal flow to the boundary conditions and a rough assessment of the process windows. Smaller parts being much cheaper facilitate additional testing with deviated boundary conditions to predict the worst-case scenario. This is very important for mass production, where all temperatures and friction can have a natural deviation of some $10 \%$. However, it has to be understood that boundary conditions are not the only possible reason for the instability of metal flow; for instance, material softening can be another one. In this case, the situation could be opposite because all volumetric effects are relatively stronger in the large-scale models, and additionally, material softening can be more significant in large forgings due to coarser initial microstructure of the material. A logical conclusion to these observations can be following: scaled down trials definitely provide plenty of useful information and an improved understanding of the parent process, but the obtained results must be scrutinized and not projected directly to the parent process. 


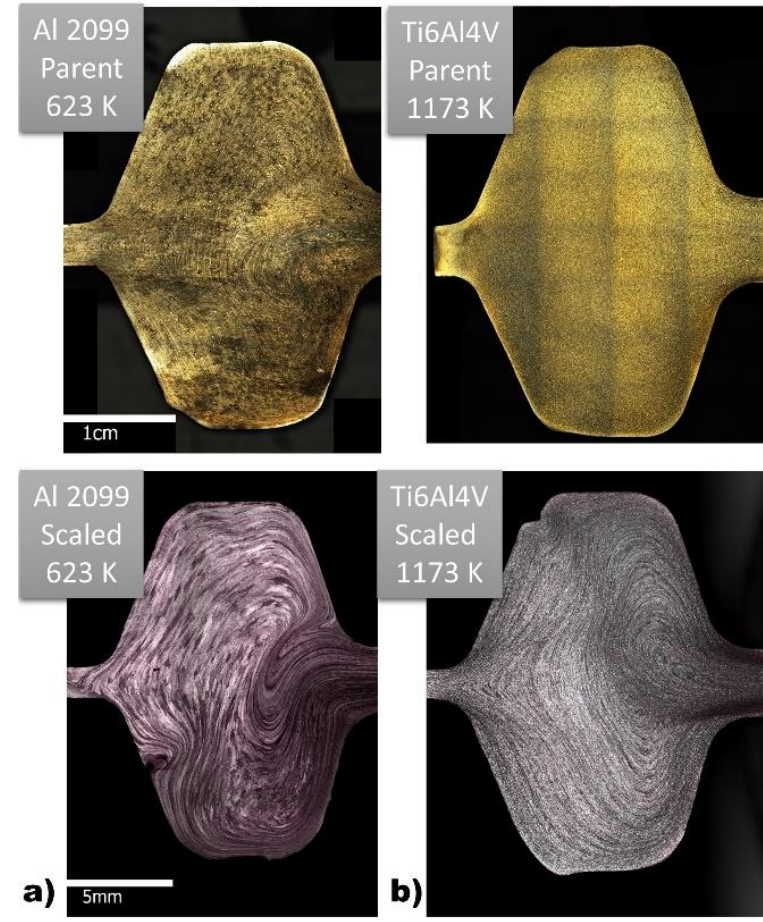

c)

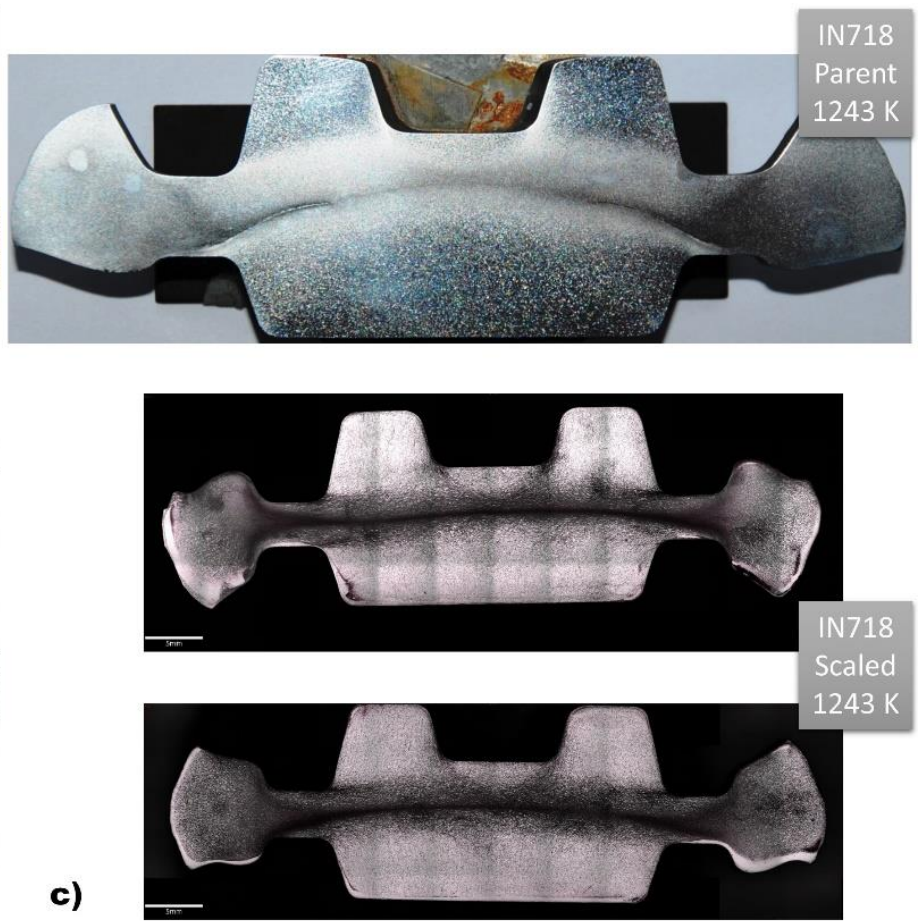

Figure 12. Illustration of the higher sensitivity of the scaled down trials for all three material - (a) AA2099, (b) Ti6A14V and (c) IN718

The remaining question discussed here is the sensitivity of different materials to scaling. It is clearly evident that the heat capacity and heat conductivity of the material along with the thermal sensitivity of the constitutive behaviour mainly define the difference in behaviour between scaled and parent forgings. The higher is the heat conductivity the greater the heat flux at the boundary between the material and dies (for the same temperature difference). The lower is the value of specific heat the bigger the volume of material exposed to temperature change for the same heat flux. The higher the thermal conductivity the smaller the temperature gradients for a given heat flux. Similarly, higher values of specific heat reduce the effect of adiabatic heating on temperature. Following this evident logic, materials with high specific heat and low thermal conductivity will be less sensitive to scaling (especially in slow forging process, where adiabatic heating is insignificant).

Among materials investigated in this paper and listed in the Table 4, Ti6Al4V has the best combination of specific heat and thermal conductivity. Metal flow in the parent and scaled down trials for this material is shown in the Fig. 13. It can be seen that inside the volume, grain flow is quite similar, and the main differences are only at the boundaries, where the boundary conditions play a major role, as discussed above. Inconel 718, due to relatively low specific heat and low conductivity, suffers from large gradients of temperature with "sharp" transitions resulting in significantly different metal flow and recrystallization processes in the scaled down models. This is the worst case among those investigated here. If scaled experiments with Nickel superalloys are essential due to some reasons, apparently, some special methodology should be developed, e.g. some compensational 
heating, especially if the scaling factor is large. Al 2099 represents the middle case, as it has an interesting combination of very high specific heat and medium thermal conductivity. The overcooled zone at the boundaries can increase the brittleness of the material, and can lead to cracking, as previously discussed, and break the similarity of metal flow. However, it may be avoided with some additional heating - for which more investigations are required.
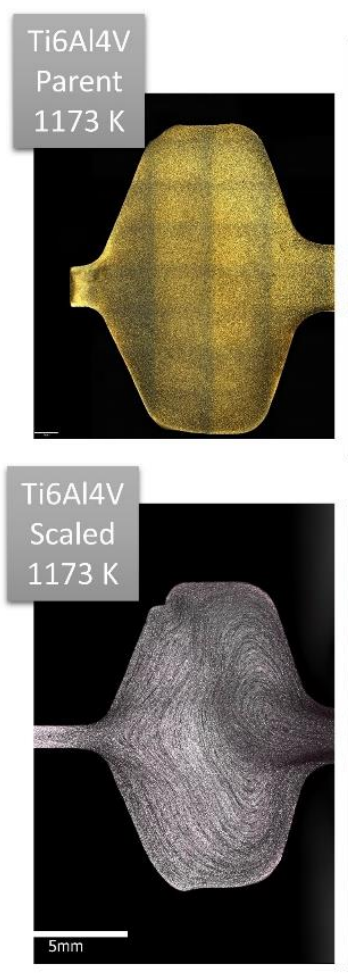
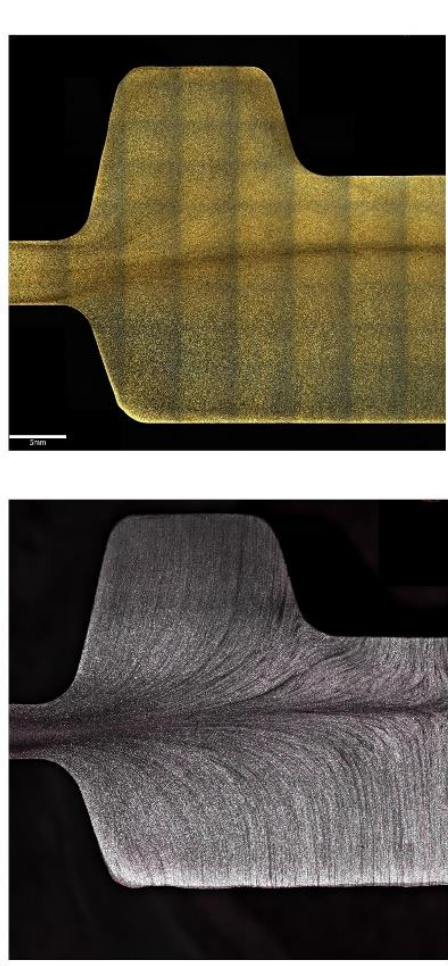
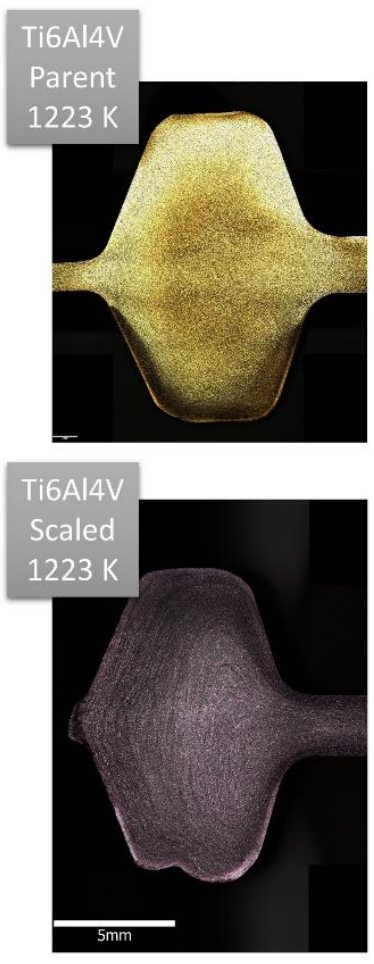
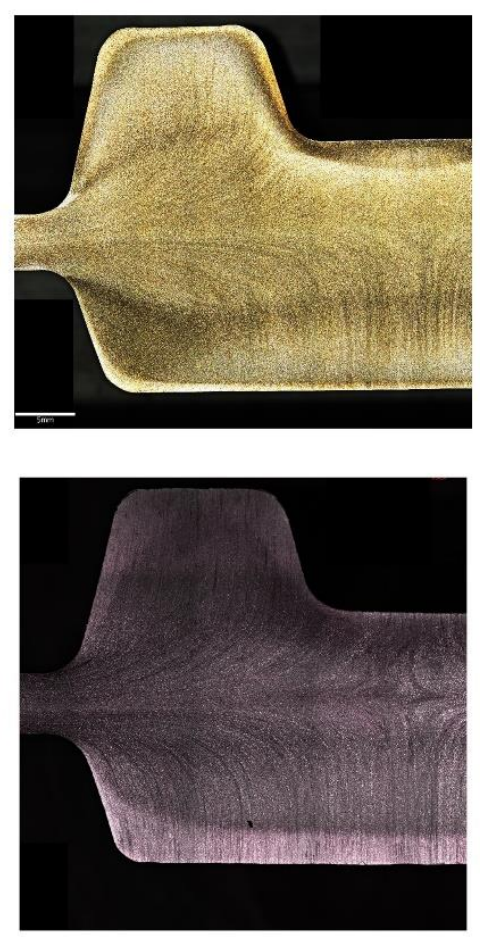

Figure 13. Illustration of the case with best similitude in the study carried out - results for inexact scaling with Ti6Al4V

\section{Conclusions}

Considered in this paper is the development and application of inexact-finite similitude for the purposes of forging scaled experiments. The following conclusions can be drawn from the presented work:

- Finite similitude has been shown to be equivalent to the removal of the length scale $\beta$ from the transport equations projected onto the physical space.

- A procedure for the formation of digital twins for the materials and forging processes has been established, which facilitates the design and analysis of scaled experimentation.

- A means for the identification of process and parameter windows has been established in the paper for substitute materials in the scaled forging experiments.

- Despite big differences in the results of parent and scaled down experiments, inexact scaling has been shown to provide useful information, significantly broadening the process understanding. 
- Scaled down inexact scaling experiments have been shown to be more sensitive than the parent large-scale ones. This can be exploited by using scaled down trials as a "sensors" to investigate the possibility of flow instability in large-scale forgings.

- In many cases, the stable processing window for scaled-down forging looks to be narrower than for the large parent ones. This confirms that in spite of the lack of exact similitude between two processes, miniaturized trials still can be useful to access the limits of industrial process window, stability of the process and range of tolerances. 


\section{Acknowledgements}

Acknowledgement and thanks are made to the EPSRC for financial support in the form of an EPSRC HVM Catapult Fellowship to enable Dr Davey to work at the Advanced Forming Research Centre (AFRC) in Glasgow UK. In addition, thanks and acknowledgements are extended to the University of Manchester and the AFRC, whom provided generous support. In particular, the University of Manchester gifted Dr Davey with sabbatical leave and academic cover and the AFRC provided Catapult money to facilitate the extensive set of experiments performed in the work. 


\section{References}

Politis, D. J., Politis, N. J., Lin, J., Dean, T. A., A review of force reduction methods in precision forging axisymmetric shapes, The International Journal of Advanced Manufacturing Technology, Vol. 97(5-8), pp. 2809-2833, (2018).

Yang T. S., Prediction of maximum forming load and billet dimensions using an abductive network and finite element method simulation of a near net-shaped helical gear forging, Proc Inst Mech Eng, Part B: J Eng Manuf, Vol. 233(3), pp. 289-301, (2009).

Chen, M.-S., Lin, Y. C., Numerical simulation and experimental verification of void evolution inside large forgings during hot working, International Journal of Plasticity, Vol. 49 pp. 53-70, (2013).

Hawryluk, M., Ziemba, J., Sadowski, P., A Review of Current and New Measurement Techniques Used in Hot Die Forging Processes, Measurement and Control, Vol. 50(3), pp. 74-86, (2017).

Gronostajski, Z, Pater, Z, Madej, L., Gontarz, A., Lisiecki, L., Łukaszek-Sołek, A., Łuksza, J., Mróz, S., Muskalski, Z., Muzykiewicz, W., Pietrzyk, M., Śliwa, R.E., Tomczak, J., Wiewiórowska, S., Winiarski, G., Zasadziński, J., Ziółkiewicz, S., Recent development trends in metal forming, Archives of Civil and Mechanical Engineering, Vol. 19, pp. 898-941, (2019).

Tian, Z., Gao, F., Jin, Z., Zhao, X., Dimension measurement of hot large forgings with a novel time-of-flight system, The International Journal of Advanced Manufacturing Technology, Vol. (44), pp. 125-132, (2009).

Zhang, F.-L., Zhang, Y.-C., Zhang, L.-Q., Hou, X.-Y., The interplay between the measured dimension and temperature of hot large forgings, The International Journal of Advanced Manufacturing Technology, Vol. 81(9-12), pp 1619-1630, (2015).

Sedov, L. I., Similarity and dimensional methods in mechanics, CRC press, (1993).

Zohuri, B., Dimensional analysis and self-similarity methods for engineers and scientists, Springer, (2015).

Barenblatt, G. I., Scaling, Cambridge University Press, Vol. 34, (2003).

Kline, S. J., Similitude and approximation theory, Springer Science \& Business Media, (2012).

Altan, T., Henning, H.-J. \& Sabroff, A. M. The use of model materials in predicting forming loads in metalworking, Journal of Engineering for Industry, Vol. 92, pp. 444-451, (1970).

Navarrete, J., Noguez, M., Ramírez, J., Salas, G. \& Robert, T., Die forging stress determination: a dimensional analysis approach, Journal of Manufacturing Science and Engineering, Vol. 123, pp. 416-419, (2001). 
Pawelski, O., Ways and limits of the theory of similarity in application to problems of physics and metal forming, Journal of Materials Processing Technology, Vol. 34, pp. 19-30, (1992).

Sofuoglu, H., Rasty, J., Flow behavior of Plasticine used in physical modeling of metal forming processes, Tribology International Vol. (33), pp. 523-529 (2000).

Eckerson, K., Liechty, B., Thermomechanical similarity between Van Aken plasticine and metals in hot-forming processes, The Journal of Strain Analysis for Engineering Design, Vol. 43(5), pp. 383-394, (2008).

Krishnamurthy, B., Bylya, O., Davey, K., Physical modelling for metal forming processes, International Conference on the Technology of Plasticity, ICTP 2017, 17-22 September 2017, Cambridge, United Kingdom, Procedia Engineering, 207, pp. 1075-1080, (2017).

Davey, K., Darvizeh, R. and Al-Tamimi, A., Scaling of Metal Forming Processes Approach, International Conference on the Technology of Plasticity, ICTP 2017, 17-22 September 2017, Cambridge, United Kingdom, Procedia Engineering, 207, pp. 1069-1074, (2017).

Casaburo, A., Petrone, G., Franco, F., De Rosa, S., A Review of Similitude Methods for Structural Engineering, Appl. Mech. Rev., 71(3), p 32, (2019).

Simitses, G. J., and Rezaeepazhand, J., Structural Similitude for Laminated Structures, Compos. Eng., Vol. 3(7-8), pp. 751-765, (1993).

Coutinho, C. P., Baptista, A. J., Rodrigues, J. D., "Reduced Scale Models Based on Similitude Theory: A Review up to 2015,” Eng. Struct., Vol. 119, pp. 81-94, (2016).

Davey, K., Darvizeh, R., Al-Tamimi, Scaled Metal forming: A transport equation approach, International Journal of Solids and Structures, 125, pp. 184-205, (2017).

Al-Tamimi, A., Darvizeh, R., Davey, K., Experimental investigation into finite similitude for metal forming processes, Journal of Materials Processing Technology, 262, pp. 622-637, (2018).

Moghaddam, M., Darvizeh, R., Davey, K., Darvizeh, A., Scaling of the powder compaction process, International Journal of Solids and Structures, 144-145, pp. 192-212, (2018).

Ochoa-Cabrero, R., Alonso-Rasgado, T., Davey, K., Scaling in biomechanical experimentation: A finite similitude approach, Journal of the Royal Society Interface, Vol. 15(143), 20180254, (2018).

Sadeghi, H., Davey, K., Darvizeh, R., Darvizeh, A., A scaled framework for strain rate sensitive structures subjected to high rate impact loading, International Journal of Impact Engineering, Vol. 125, pp. 229-245, (2019a).

Sadeghi, H., Davey, K., Darvizeh, R., Darvizeh, A., Scaled models for failure under impact 
loading, International Journal of Impact Engineering, Vol. 129, pp. 35-56, (2019b).

Lin, J., Selection of material models for predicting necking in superplastic forming, International Journal of Plasticity, Vol. 19(4), pp. 469-481, (2003).

Alaborta, E., Putman, D., Reed, R. C., Superplasticity in Ti-6Al-4V: Characterisation, modelling and applications, Acta Materialia, Vol. 95(15), pp. 428-442, (2015).

Padmanabhan, K.A., Vasin, R.A., Enikeev, F.U., Superplastic Flow: Phenomenology and Mechanics, Springer-Verlag Berlin Heidelberg, (2001)

Prasad, Y.V.R.K., Rao, K.P., Sasidhara, S., Hot Working Guide: A Compendium of Processing Maps, Second Edition, ASM International, (2015)

Zhang, F., Sun, J. L., Shen, J., Yan, X. D., Chen, J., Flow behavior and processing maps of 2099 alloy, Material Science Engineering A, 613, pp. 141-147, (2014).

ASTM International. ASTM E8/E8M-16a Standard Test Methods for Tension Testing of Metallic Materials. West Conshohocken, PA; ASTM International, (2016). doi: https://doi.org/10.1520/E0008_E0008M-16A.

ASTM International. ASTM E21-17e1 Standard Test Methods for Elevated Temperature Tension Tests of Metallic Materials. West Conshohocken, PA; ASTM International, (2017). doi: https://doi.org/10.1520/E0021-17E01.

F. Zhang, J. L. Sun, J. Shen, X. D. Yan, J. Chen, Flow behaviour and processing maps of 2099 alloy, Mater. Sci. Eng. A, 613 (2014) 141-147 


\section{Appendix}
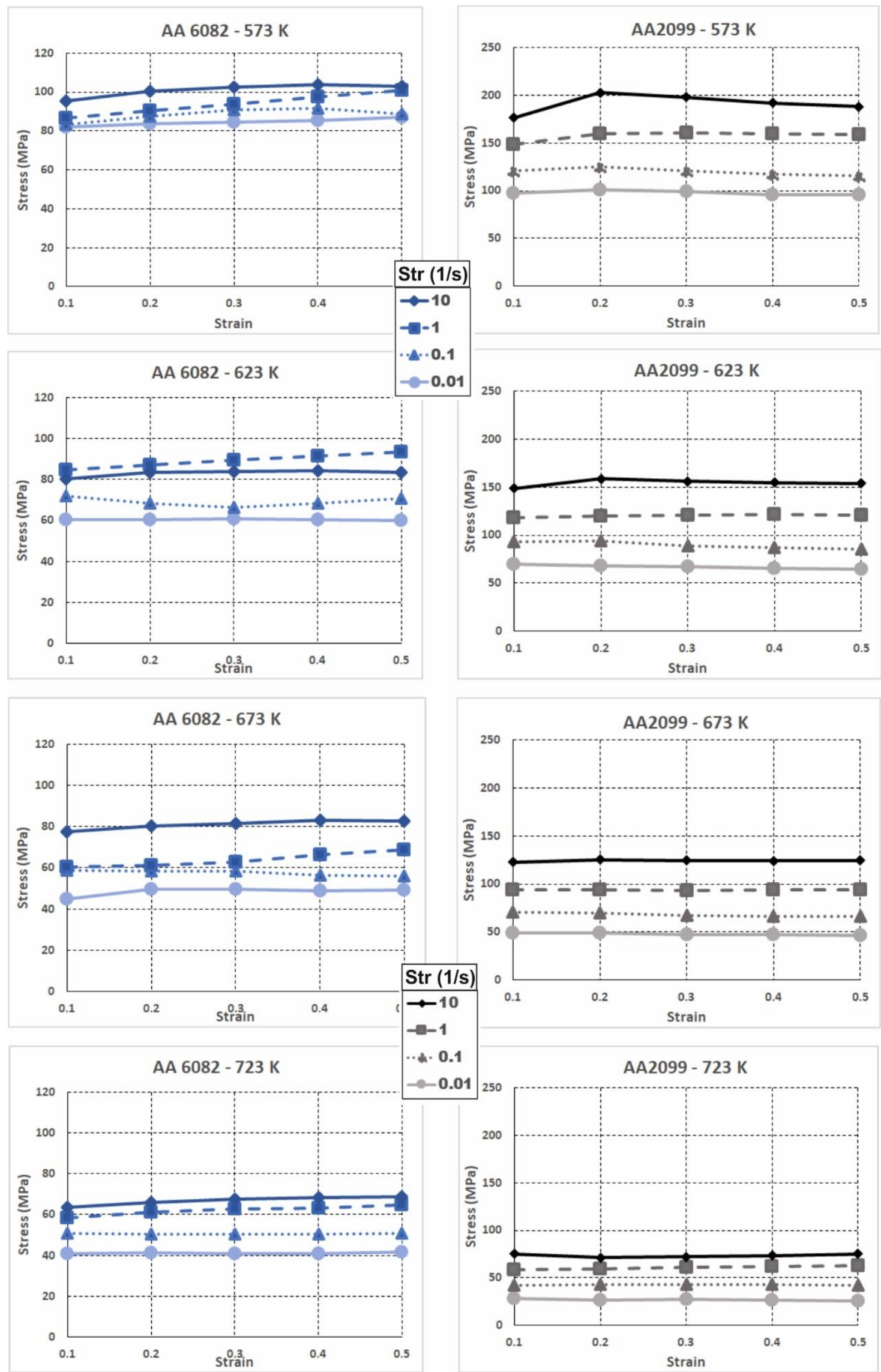

Figure 14. Flow stress data for AA2099 and AA6082 alloy obtained from literature (Prasad et al., 2015, Zhang et al., 2014) and used in the present study 

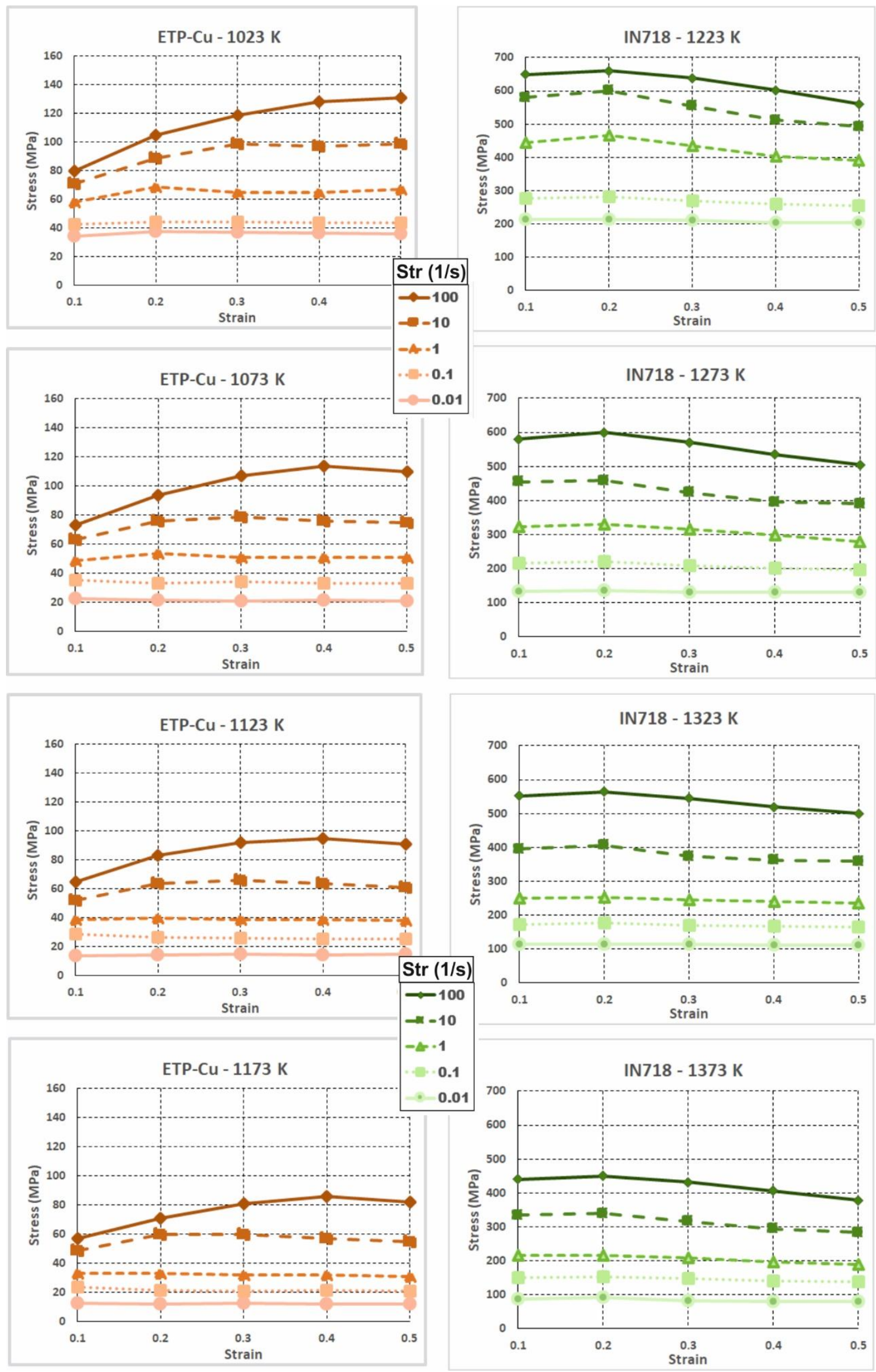

Figure 15. Flow stress data for ETP-Cu and IN718 alloy obtained from literature (Prasad et al., 2015) and used in the present study 

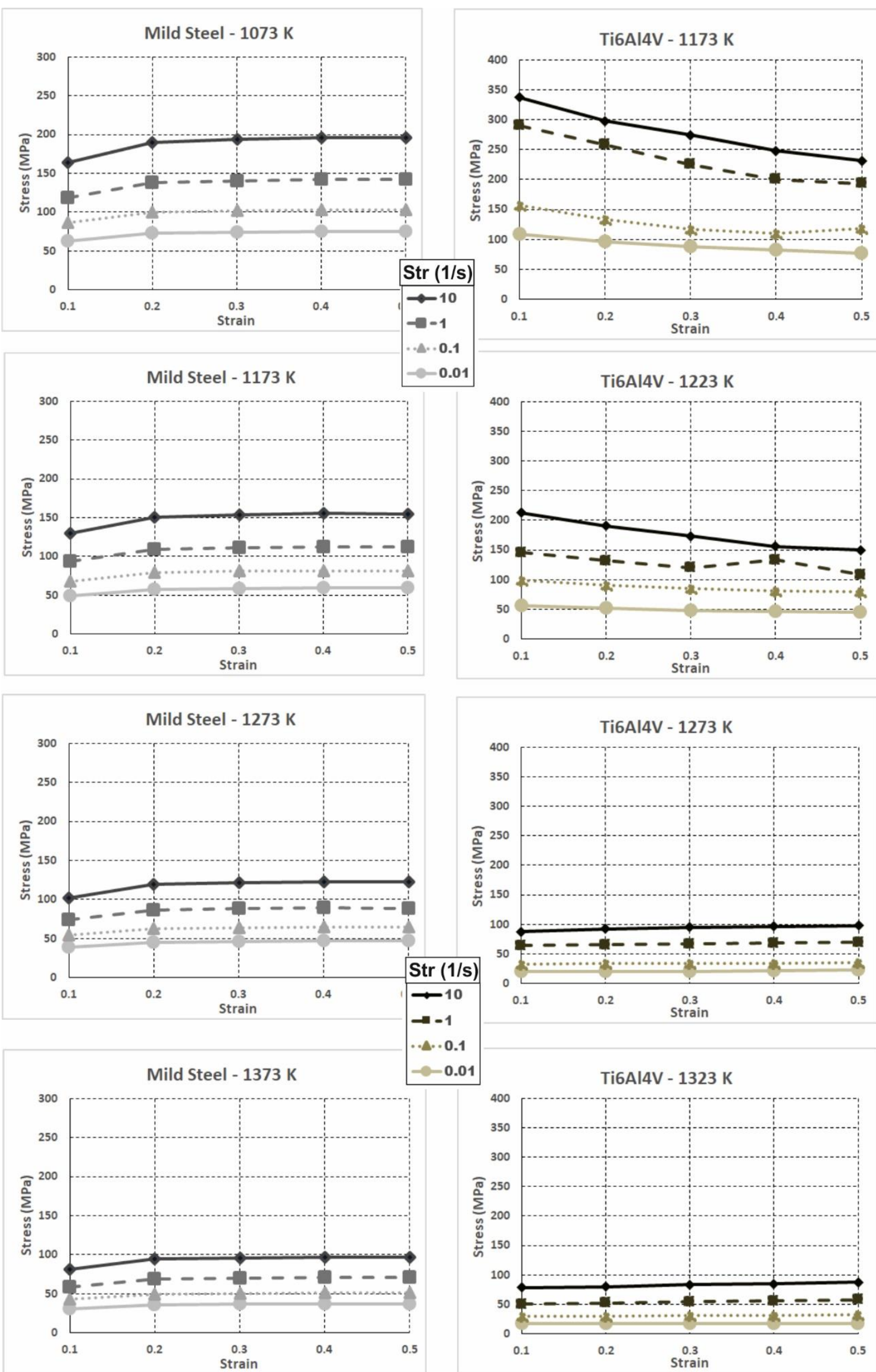

Ti6Al4V - $1323 \mathrm{~K}$

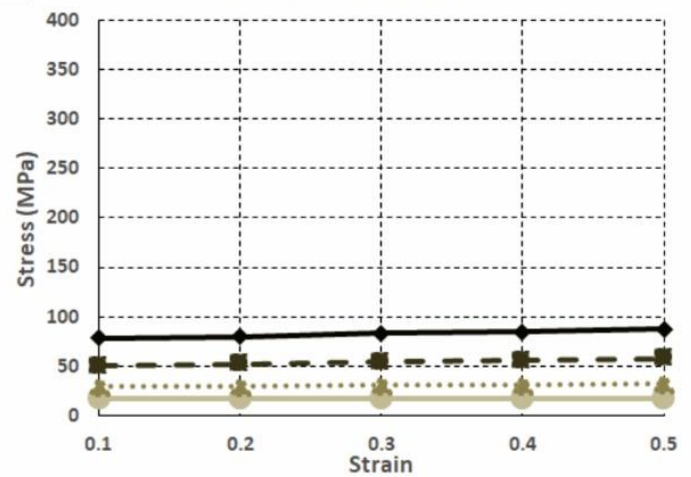

Figure 16. Flow stress data for Mild Steel and Ti6Al4V alloy obtained from literature (Prasad et al., 2015) and used in the present study 